\title{
Blockchain and COVID-19 Pandemic: Applications and Challenges
}

This paper was downloaded from TechRxiv (https://www.techrxiv.org).

\section{LICENSE}

CC BY 4.0

SUBMISSION DATE / POSTED DATE

10-09-2020 / 17-09-2020

\section{CITATION}

Ahmad, Raja Wasim; Salah, Khaled; Jayaraman, Raja; Yaqoob, Ibrar; Ellahham, Samer; Omar, Mohammed (2020): Blockchain and COVID-19 Pandemic: Applications and Challenges. TechRxiv. Preprint. https://doi.org/10.36227/techrxiv.12936572.v1

DOI

10.36227/techrxiv.12936572.v1 


\title{
Blockchain and COVID-19 Pandemic: Applications and Challenges
}

\author{
Raja Wasim Ahmad, Khaled Salah, Raja Jayaraman, Ibrar Yaqoob, Samer Ellahham, and Mohammed Omar
}

\begin{abstract}
The year 2020 has witnessed the emergence of coronavirus disease (COVID-19) that has rapidly spread and adversely affected the global economy, health, and human lives. The COVID-19 pandemic has exposed the limitations of existing healthcare systems regarding its inadequacy to timely and efficiently handle public health emergencies. A large portion of today's healthcare systems are centralized and fall short in providing necessary information security and privacy, data immutability, transparency, and traceability features to detect frauds related to COVID-19 vaccination certification, anti-body testing, and medical supplies. Blockchain technology can assist to combat the COVID-19 pandemic by assuring safe and reliable medical supplies, accurate identification of virus hot spots, and establishing data provenance to verify the genuine personal protective equipment that is decentralized, trustworthy, traceable, and transparent. In this paper, we discuss the potential blockchain applications for the COVID-19 pandemic. We present the high-level design of three blockchain-based systems to enable the governments and medical professionals to efficiently handle health emergencies caused by COVID-19. We discuss the important ongoing blockchain-based research projects to demonstrate the adoption of blockchain technology for the COVID-19. Finally, we identify and discuss future research challenges along with their key causes and guidelines.
\end{abstract}

Index Terms-COVID-19, Blockchain, Healthcare, Traceability, Security.

\section{INTRODUCTION}

Coronavirus disease (COVID-19) is a respiratory infection that has globally affected various sectors such as economy, healthcare, transportation, education, to name a few. COVID19 is dangerous particularly for people with pre-existing medical conditions and can quickly spread from humans to humans [1-3]. It is estimated that COVID-19 will reduce global economic growth by $3 \%$ to $6 \%$ in 2020 [4]. The COVID-19 has spread beyond 213 countries and independent territories, with over 19,462,112 people infected, and death toll has reached 722,285 [5]. Health agencies such as the world health organization (WHO) have recommended several protective measures to immediately respond and limit the unprecedented global spread of COVID-19. The scarcity of medical supplies and hospital capacity has forced government authorities to impose partial or complete lockdown to contain the spread of the infection. Prevention from the adversarial effects due to the spread of COVID-19 requires coordinated action and

R. W. Ahmad, K. Salah, R. Jayaraman, I. Yaqoob, and M. Omar are with the Research Center on Digital Supply Chain and Operations Management (DSO), Khalifa University of Science and Technology, Abu Dhabi 127788 , UAE.

Samer Ellahham is associated with Heart \& Vascular Institute, Cleveland Clinic Abu Dhabi, UAE.

Corresponding author: ibraryaqoob@ieee.org collaboration among the health professionals, authorities, research institutes, and government $[1,2,6]$. However, the legacy information management systems being used to store crucial COVID-19 related data are mostly disintegrated. Disintegrated systems suffer from a lack of adequate means to share data and can create information silos for participating organizations. Information silos can minimize collaboration opportunities among participating organizations to combat the COVID-19 pandemic. The use of technologies such as blockchain can assist business organizations to minimize the adverse effects of the COVID-19 pandemic. The inherent features of blockchain technology can foster information sharing and present a unified view of data to improve the coordination and actions of organizations to minimize the spreading of COVID-19.

Recently, numerous technology-based applications have been developed worldwide to assist authorities to closely monitor public health to combat the COVID-19 pandemic. For example, many corporate sectors such as Google and Apple have recently launched contact tracing applications that can help authorities to trace COVID-19 infected persons. But, the existing solutions have to access personal data such as location and COVID-19 test results of an individual to identify the spread rate and predict viral hotspots within a community [7-9]. A large portion of the proposed systems has followed centralized architecture to access, store services, and data related to COVID-19. For instance, Singapore's contact tracing solution called TraceTogether, employs Bluetooth technology to discover the close contact of a person with an infected patient with COVID-19 $[8,10,11]$. Being a centralized governed solution, the contact tracing service providers can access user data to compromise data privacy. Similarly, the data records and transactions in centralized-based systems are vulnerable to modifications, fraud or deletion. Also, centralized systems are less trustworthy as they are prone to a single point of failure $[12,13]$. They can offer limited opportunities for collaboration among organizations including healthcare, government, and law-enforcement agencies. Also, centralized systems fall short to provide complete traceability, transparency, and immutability of data stored and exchanged during various operational processes to deal with the COVID-19 pandemic.

Blockchain is a distributed, decentralized, and immutable record of transactions that are stored on a distributed network of nodes over geographically dispersed locations [6]. The decentralization feature of blockchain technology provides high security and robustness to the data and transactions stored on the blockchain with no possibilities of a single point of failure attack. The record of transactions and data stored on the blockchain is transparent to each member in the network 
that brings trust in reliability and availability of data [6, 1416]. The transparency of blockchain-based records is achieved through a decentralized consensus protocol that defines rules for assuring the agreement of all miners on the current status of blockchain. The miner nodes in the blockchain network validate the new transactions and store them as a new block to the existing blockchain ledger. Miners are usually rewarded with cryptocurrencies for their mining services. For instance, proof-of-work (PoW) $[16,17]$ consensus protocol assures that a miner uses its computational power to solve a cryptographic puzzle for mining a block. The integrity of transactions on the blockchain network is assured through hashing algorithms and asymmetric cryptography. Blockchain uses asymmetric cryptography to validate the authenticity and integrity of data. Blockchain technology employs hashing (a cryptographic algorithm) to link each block with the predecessor to make the data on the blockchain immutable. In general, blockchain platforms are categorized as permissionless or permissioned blockchain. Any user can join, make transactions, and involve in the mining process on the permissionless blockchain platform [6, 18]. Nevertheless, permissioned blockchain [19] is an invitation-only network that is usually managed by a single organization. Access privileges to transact on a permissioned blockchain are limited to members of the organization only $[6,15,16,20,21]$.

Blockchain technology can employ smart contracts to automate business processes and resolve disputes among healthcare collaborators in a reliable and trusted way. A smart contract is a self-executing program that establishes the trust among the participating organizations [9, 22]. For instance, in a blockchain-based system used for logistic supply chain management of COVID-19 polymerase chain reaction (PCR) testing kits, smart contracts can play a pivotal role to (a) track the location of shipping containers of testing kits, (b) identify flawed testing kits, (c) monitor the state of testing kits during their shipment, (d) and allow government officials to access data to analyze demand and supply of testing kits in a particular area. Moreover, smart contracts can simplify enrollment, execution, and managerial processes related to vaccine trial tracking, coronavirus disease diagnosis, COVID19 hotspots identification, COVID-19 outbreak tracking, and user data privacy assurance through registration services of premissioned blockchain platforms [23]. Since the outbreak of COVID-19, the spread of misinformation and rumors (e.g., vaccine development news) about COVID-19 is being reported in several countries. Smart contracts can assist to identify, verify, and prevent the spread of misinformation about COVID-19 [24]. To date, there exist a few surveys that have explored the pivotal role of blockchain technology to combat the COVID19 pandemic. For instance, the work presented in [2] has explored several technologies such as the Internet of things (IoT), 5G, drones, Artificial intelligence (AI), and blockchain, to discuss what role these technologies can play to combat the COVID-19 pandemic. In [23], researchers studied the possible benefits of integrating AI with blockchain technology to minimize the spreading of COVID-19. Nadeem et al. [8] surveyed existing digital contact tracing schemes to combat the COVID-19 pandemic. Unlike the aforementioned surveys, our work is comprehensively focused on health emergency services. The main contributions of this paper include:

- We discuss the potential blockchain applications for the COVID-19 pandemic primarily from the public health emergency perspective. We further explore each identified opportunity by demonstrating its key role using multiple use case scenarios.

- We present the high-level design of blockchain-based systems for COVID-19 data tracking, digital medical passports, and digital contact tracing to highlight their system-level components, participants, and roles definition.

- We provide insightful discussions on recent ongoing research projects to show the practicality of blockchain technology in different domains for implementing healthcare services to minimize the spread of COVID-19.

- We identify and discuss several key open research challenges that hinder blockchain technology to fully realize its potential to combat the COVID-19 pandemic.

The remainder of the paper is organized as follows. Section II explores the potential opportunities offered by blockchain technology to combat the COVID-19 pandemic and illustrates several use cases and applications. Section III reviews recently reported blockchain-based research projects for combating the COVID-19 pandemic. Section IV presents a discussion on research challenges associated with the use of blockchain to combat the COVID-19 pandemic. Section V discusses the conclusions and potential opportunities for future research.

\section{ApPlications OF BlockChain FOR FIGHTING COVID-19}

Blockchain can assist in building a transparent and efficient system (e.g., healthcare) to combat the COVID-19 pandemic through trusted, verified, distributed, and tamperresilient ledger technology. It can create the first line of defense through a network of connected devices. In this section, we explore the potential applications and use cases that blockchain technology can provide to deal with the COVID-19 pandemic. Fig. 1 outlines the key services requiring high operational transparency, data provenance, privacy, and security to combat the COVID-19 pandemic.

\section{A. Track and Trace of Personal Protective Equipment}

The use of personal protective equipment (PPE) by individuals having exposure to transmittable disease (e.g., COVID-19) can greatly prevent and control the spread of the virus. For instance, during the health emergency caused by COVID-19, the use of PPEs can lead to minimize the exposure of front line health professionals to the infected persons. Examples of PPEs that are primarily used to prevent contact to infected persons or surfaces include gloves, safety goggles, footwears, face masks, helmets, and protective clothing [25, 26]. During the COVID-19 pandemic, many counties have reported the shortage of PPEs in hospitals due to a lack of a trusted system to present accurate data about demand and supply of PPEs. In certain cases, the limited supply of PPEs against its suddenly increased demand in the health sector forced 
medical professionals to use tape to patch-up tore masks to prevent contact with COVID-19 [3, 26-29]. Several countries and organizations have experienced the supply of low-quality PPEs including face masks. One of the reasons for shipment of the low-quality PPE is the limited transparency in the logistics supply chain management process. The existing centralizedbased PPE supply chain management systems are inherently incapable to efficiently trace the data provenance of the PPEs in a trusted and reliable manner. Therefore, determining the source of PPEs along with additional details such as the type of certification of the PPE is challenging.

The blockchain $[28,30]$ adaptability by the relevant healthcare organizations to control and manage the supply chain of PPE can greatly assist in identifying PPE related frauds. It can help in building a more resilient supply chain of PPE [31]. Through blockchain-based systems, the participating organizations can verify the authenticity of PPE and identify any sign of PPE tampering or inadequate handling during its shipment. Blockchain technology immutably and transparently store all movements, ownership details, and modifications that are happened to the PPE in a distributed ledger. Immutable logs of transactions that are performed by participating organizations support auditability and provenance of PPE. Blockchain can assist to (a) secure supply chain operations and PPE certificates, (b) prevent compliance violation, (c) identify counterfeit PPEs through data provenance, (d) allow verifiable collateral-based payment settlements, (e) impose penalties to individuals for any failure of compliance with safety measures, and (f) procure PPEs from the reputed, trusted, and certified manufacturers. Moreover, smart contracts that are programmed for managing access control and automation can assist governments, authorities, and medical companies to track and trace (in a real-time) the PPE to forecast demand. The PPE traceability supported demand forecasting (using AI techniques) can be helpful to better allocate and reallocate the available PPEs. Moreover, through registered and authorized sensors, data can be collected about the available stock in the inventory and smart contracts can automatically trigger notifications for the procurement managers to place an order for more PPEs to prevent possible consequences of PPE shortage.

\section{B. Logistics Monitoring of Vaccine}

To curb the spread of COVID-19 requires successful and indispensable immunization of humans against the virus through the administration of an active vaccine. At the time of writing of this paper, many research institutes and laboratories are in the process of conducting clinical trials of several vaccine candidates. The effectiveness, safety, and genuinity of the vaccine are of great concern to the authorities, governments, and research institutes as the newly administered vaccine might adversely affect the health of an individual [30, 32-34]. The current centralized-based vaccine management systems face several challenges related to the threat of being failing to successfully secure and distribute vaccines, and breaching the logistics supply chain of vaccines for malicious purposes. Fake pharmaceutical companies consider this limitation of technology as an opportunity to sell and distribute fake and counterfeit vaccines to cure COVID-19 patients. A fake, counterfeit, or substandard vaccine is mainly manufactured using substandard material $[35,36]$. Employing poor manufacturing practices during the development of a vaccine also result in substandard vaccines. The infiltration of fake, counterfeit, or substandard vaccines into the grey market can harm human lives. For instance, due to limited operational transparency, the adversaries can successfully forge vaccine expiration or production data during its shipment or retail to increase profit.

Blockchain technology [28] can permanently store data related to various stages, phases, and events of the COVID19 vaccine such as (a) development, (b) production, (c) certification, and (c) allotment to authorized organizations for immunization purpose. In hospitals, medical professionals can access blockchain to identify, trace, and verify vaccines data before administering it. It can also be used for notification management purposes (real-time) through lightweight smart contracts. Smart contracts provide opportunities to detect vaccine-related frauds, assure zero downtime, and eliminates the role of third-party services to monitor COVID-19 vaccine logistics. The immutability feature assures that the details about the vaccine cannot be modified or deleted by the adversaries. Smart contracts can identify and verify the expiry date of the vaccine in a trusted manner using records such as the manufacturing date and warranty period of the vaccine. Also, smart contracts can use provenance data to identify the substandard and falsified vaccines manufactured and shipped through unauthorized manufacturers. For supply chain logistics services, smart contracts can be configured to monitor the state of the container for temperature, humidity, pressure, and other indices to protect the COVID-19 vaccine during its shipment $[31,37]$. The smart contracts can automatically notify the relevant authorities when the preassigned conditions for the shipment are violated. The sensors can further assist to identify any illegal attempts that may disrupt the state of the packages carrying vaccines inside the shipping container. Any such activity can be recorded, audited to monitor noncompliance, and notified in real-time to the relevant authority. The other advantages of blockchain for logistics of the future vaccine for COVID-19 include (a) transaction settlement, (b) audit transparency, (c) accurate costing information, (d) automation, (e) reducing human errors, and (f) enforcing tariff and trade policies.

\section{Incentive-Based Volunteer Participation in Clinical Trials}

Conducting clinical trials to develop the COVID-19 vaccine is a complex, time consuming, and costly process. It requires close coordination and collaboration among organizations that are involved in clinical trials of vaccine and they are often located at geographically distributed locations. Researchers, regularities, donors, and pharmaceutical companies are examples of the organizations that are actively involved in the clinical trials to successfully develop and administer the vaccine for COVID-19. The conventional centralized-based clinical trial data management systems face several challenges which are mainly related to the subject enrollment, limited performance 


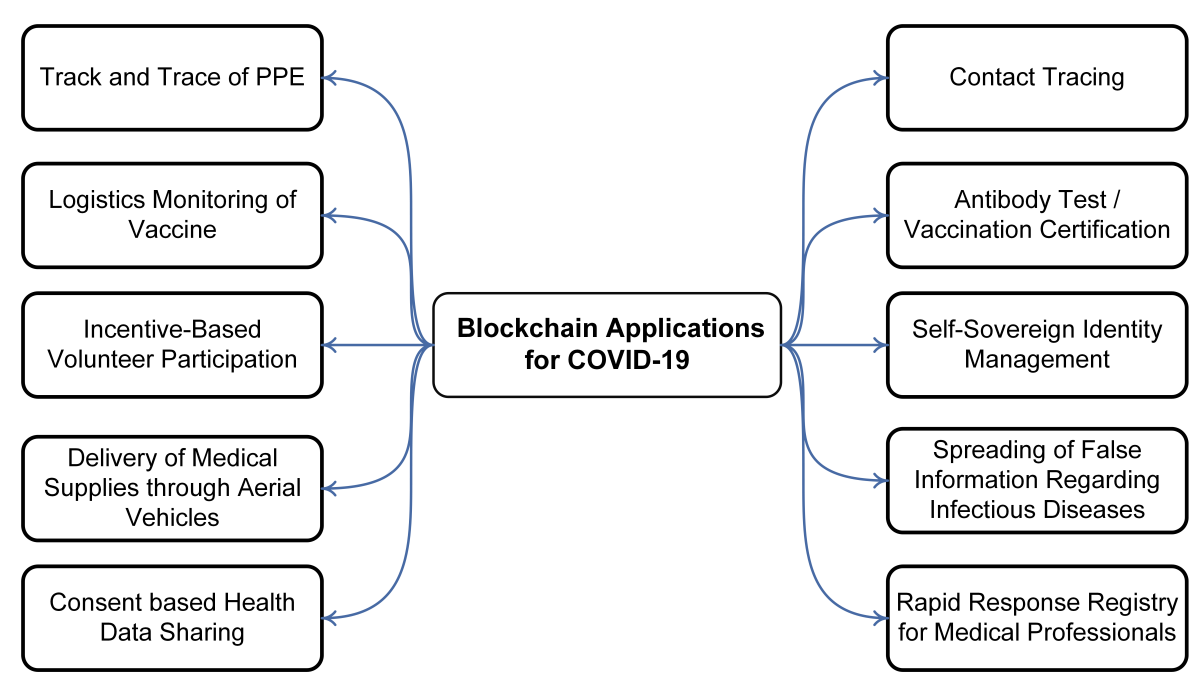

Fig. 1. Opportunities for Blockchain to combat COVID-19 pandemic.

and non-compliance with the clinical trial requirements, data privacy assurance, compliance with clinical trial rules for the health safety of participants, and integrity of clinical trial data [9, 38-41]. Also, the centralized-based clinical trial management systems can present several versions of clinical trial data that can create information silos of organizations. As a result, it can lead to duplicated clinical trial data that is often stored and managed by multiple organizations. Thus, duplication of clinical trial data makes it difficult to access, process, and analyze results. Also, centralization makes clinical trial data vulnerable to modifications by external hackers or participants.

Blockchain technology can enable pharmaceutical companies and research institutes to preserve the integrity of clinical trial data during the development of a vaccine. It assures that a single and synchronized view of clinical trial data is available for all authorized organizations. Thus, it can successfully overcome issues such as clinical trial data duplication and inconsistency due to the disintegration of the existing centralized-based clinical trial management systems. The smart contracts can verify the access rights of an organization before permitting it to use clinical trial data for preserving data privacy and security. For compliance with clinical trial requirements, smart contracts can verify that the authorized clinical trial participants have digitally signed the consent form $[38,40]$ before triggering a transaction to read or write health data on the ledger. Therefore, an anonymized data collection and verifiable consent management can enable participants to share their case records with the authorized organizations without disclosing their identities. To retain the participants of the clinical trial, medicine companies usually offer tokens of appreciation to the participants in the form of cash or gift cards [42]. Smart contracts can assist to speed up the payment process by providing an automated, transparent, and accountable mode for transferring cryptocurrencies. The transparency and accountability features also assure that the data can be used only for the purpose for which it is collected; thereby it increases the trust of the users.

\section{Delivery of Remote Healthcare and Medical Supplies}

Leveraging advanced remote health practices such as telehealth and telemedicine services to minimize the transmission risk of contagious viruses can enable symptomatic patients to remotely communicate with health specialists through IT infrastructure. Remote diagnosis and treatment of patients can significantly minimize patient access and workforce limitations, and thus the employability of remote health services can effectively control and limit the rapid increase in global COVID-19 cases [2, 43, 44]. Being governed and managed by a centralized authority, remote healthcare systems are vulnerable to a single point of failure problem, which ultimately affects the integrity and trustworthiness of the health records. The inherent features of revolutionary blockchain technology can bring diverse benefits to the remote healthcare industry [23, 45-47]. The primary benefits include establishing the provenance of electronic health records, verifying the legitimacy of users demanding patient data, assuring patient anonymity, and automating micropayments for using remote health services. The traceability feature assists successfully establish the provenance of self-testing medical kits for COVID19 testing. Following the testing outcome, individuals whose test results are negative are usually obliged to follow selfquarantine policies to mitigate the spread of the virus to society. The requirements of secure track and trace of medical supplies for self-quarantined individuals bring about opportunities for blockchain technology to transparently store timestamped location data of medical supplies on the ledger.

Ensuring social distancing and wearing face masks during performing business activities (e.g., relevant healthcare participants) can assist in curbing the spread of COVID-19. The globally increasing COVID-19 confirmed cases demand contactless delivery of medicines to the patients especially in areas of very high virus transmission rate to further prevent COVID19 from spreading. For this purpose, aerial vehicles can be used to deliver medicines and medical supplies to remote patients. Aerial vehicles can also assist in transporting medical supplies among hospitals that are housed at distant locations. 


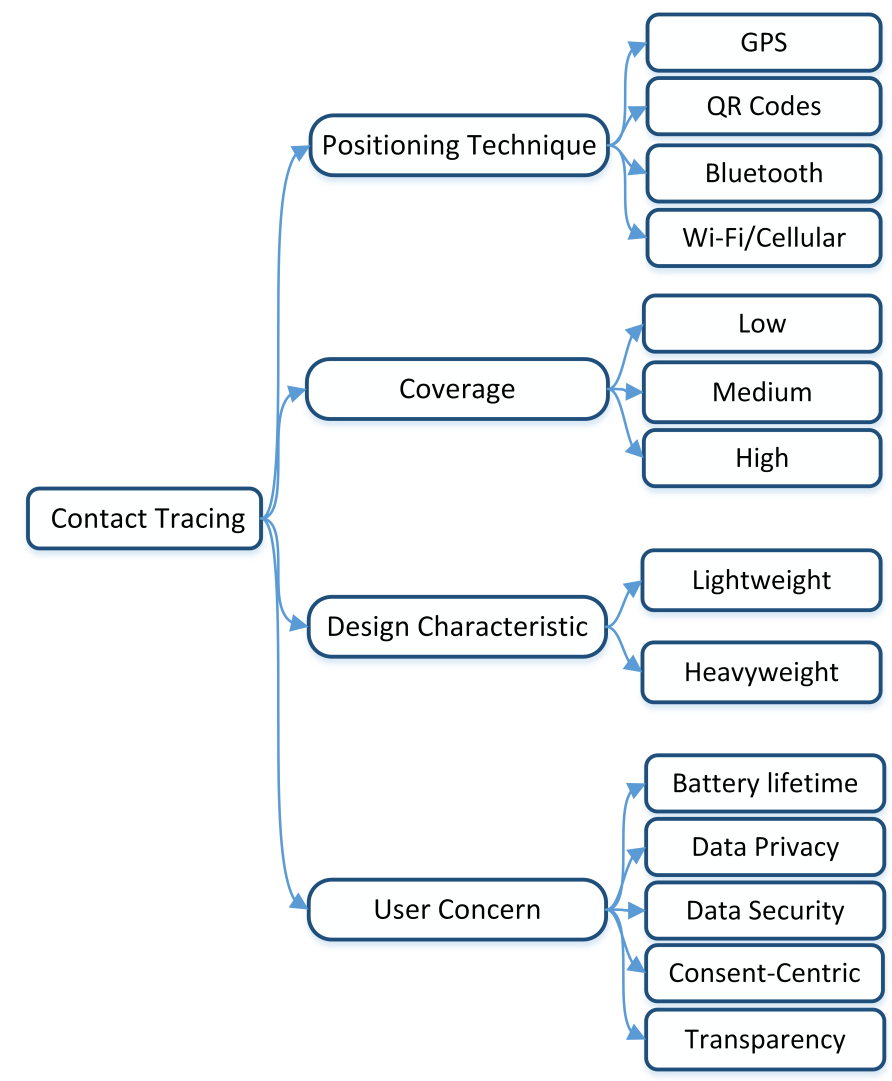

Fig. 2. Design parameters for digital contact tracing solutions.

For instance, China experimented (in 2020) employing aerial vehicles to supply medicines from one city to another during the COVID-19 pandemic [47-49]. Blockchain technology can assist to track and trace the location of the aerial vehicles, verify provisioned service level, and calculate the reputation score of an aerial vehicle based on its performance in a trusted, accountable, and transparent manner. Through implementing access control protocols and identity management, blockchain technology minimizes the possibility of attacks by the adversarial vehicles. It immutably stores commands that are issued to the aerial vehicles (for audit purposes to verify non-compliance with issued commands) by the control room along with actions to sanitize the highly virus-infected areas and detect human movements and interactions. A swarm is comprised of multiple autonomous aerial vehicles that work together to achieve a common goal. Blockchain technology can be used by the swarm of aerial vehicles to reach a highly reliable global decision by securely transacting on the blockchain. For instance, through a blockchain-based voting system, aerial vehicles of a swarm can identify the most densely populated public places to spray disinfection [2, 49].

\section{E. Digital Contact Tracing}

Respecting social distancing directives issued by the government can significantly minimize the social interactions of people to prevent the spread of COVID-19. Social distancing is implemented through a public health measure called digital contract tracing that can break the chain of the person to person transmission of the virus. Digital contact tracing continuously monitors infected people to rapidly and effectively identify all social interactions that happened during the infectious incubation period of COVID-19 infected patients. It mainly employs GPS or Bluetooth to use proximity data to identify social interaction with a virus-infected individual. After encountering a close contact with a confirmed COVID-19 case, the exposed individuals are required to be tested, monitored, and self-quarantined [50,51]. Transparency and immutability of data assure that the health data of users such as the COVID19 test result cannot be altered or deleted by the adversaries or healthcare collaborators. Also, it preserves the privacy of users data to comply with privacy rules as stated in general data protection regulation (GDPR) privacy laws [50-59]. The design parameters necessary to design and implement a contact tracking solution for identifying social interactions among people are highlighted in Fig. 2. The positioning technique parameter states the technologies that can be used to identify the locations of a user. The coverage area parameter defines the range of geographic areas within which a social interaction of a COVID-19 patient with another person can be traced. The heavyweight designs of a contact tracing application intend to use system resources aggressively during identification and verification of social interaction among people. On the other hand, the lightweight application design optimizes system resources by guiding users and provides the most important and needed features only. The requirements of digital contact tracing users include extended battery lifetime of devices and high privacy, security, and transparency of COVID-19 related data. Ideally, a digital contact tracing solution should provide high privacy of data, extended coverage area, lightweight application design, high data security and transparency, and battery-friendly operations.

Assuring the privacy of personal health data of an individual along with the occurrence of minimal COVID-19 false-positive is the key challenge for the digital contact tracing solutions. The privacy of the data is preserved by encrypting the location and contact history of a person and preventing the disclosure of personal health data to the public [60]. On encountering a close contact with a COVID-19 infected patient, users can be informed about the recent social interaction without disclosing the credentials of the COVID-19 infected individual (e.g., name). Digital contact tracing through smartphone applications such as Singapore's TraceTogether and Google-Apple's contact tracing employs Bluetooth to identify the close physical contact of a person with a virus-infected individual. But, considering the battery constraints of smartphones, TraceTogether [52] is not user friendly. Google/Apple Contact Tracing [61] does not disclose the identity and location of the users and thereby preserves data privacy. Considering the high privacy and sensitivity of users' data, the non-blockchain-based solutions are less trustworthy as they are vulnerable to data forging by the administrator of the application. The immutable and decentralized blockchain technology is a viable alternative for digital contact tracing. It preserves the privacy of the user's data by enabling pseudo-anonymity. The digital contact tracing through a regular expression matching technique can employ the blockchain platform to store social interaction data and 
allow only authorized users to access data to preserve its privacy (using consent form) [62].

Fig. 3 presents a digital contact tracing system that can be used by any organization to ensure safe distancing among its employees to restrain the virus spreading. In the presented system, an external trusted network of the servers is used to generate anonymous addresses for the users to preserve data privacy. The system has implemented many smart contracts such as Entities registration, Geodata processor, COVID-19 testing, Query processing, and Consent management to automate services and assures that credentials about the individuals who are infected from COVID-19 are not disclosed to others. Being a private blockchain-based system, all entities are registered before making a transaction onto the blockchain. Geodata processor contract assures that the duplicate data (e.g., location data of a user with limited mobility) is not forwarded to the contact solver to speed up the contact tracing process. COVID-19 testing contract assists to record COVID19 test results on the blockchain for each employee. The consent management contract seeks to legalize the location data usage of employees of an organization. The contact solver component of the contact tracing system leverages AIbased techniques for identifying social interactions among individuals $[10,63]$. It intimates the users about possible risk levels based on many factors such as distance, mobility, and total time spent during social interaction with a COVID-19 infected person.

\section{F. Vaccination Certificates and Immunity Passports}

Antibody testing, also called serology testing, specifies that a person has developed immunity against virus (e.g., COVID-19) after full recovery from the virus. The vaccination certificate enlists the diseases that a person has been vaccinated against. Vaccination certificate assists in preventing and controlling the spread of COVID-19 by enabling the authorities and governments to formulate policies by allowing cross-border traveling for those people who possess this certificate [64]. Therefore, the certificate's forgery protection, high cost-effectiveness, and privacy assurance are the key requirements of the authorities to minimize traveling related frauds. Blockchain-based antibody testing and vaccine certification provides a robust and secure data management system that is easy to administer, unforgeable, and cost-effective [6569]. Blockchain employs asymmetric encryption/decryption schemes [70] and digital signatures to protect antibody testing and vaccine certification data. Also, the decentralization feature assures protection of vaccine certificates against single point of failure or other malicious attacks, thereby it increases the trust of the users by improving data reliability and security.

The certificate of antibodies can be verified in a trusted way along with assuring the privacy of the user data. For instance, at reopening the business places after the COVID19 pandemic is over, many organizations can formulate and follow the policies to permit only those employees to return to work who possess a valid digital immunity passport (based on antibody testing and vaccination). In such a case, blockchain technology assures that due to the immutability feature an invalid immunity passport can not be presented to the authorities to access the workplace. The intrinsic transparency and traceability features of blockchain assist in establishing the data provenance of the COVID-19 lab results (through data provided by certification authorities). It can further assist the organizations to verify the legitimacy of the PCR testing kits that are used for COVID-19 testing. The key organizations or participants that could be involved in the antibody testing and vaccine certification use cases include employees, hospitals, and employers [65, 69, 71]. The hospitals/test centers (Immunity passport issuer) take the blood or swap sample to conduct the antibody testing. It creates a digital passport for the user to immutably record it on the ledger. On the other hand, the employers (Immunity passport verifier) can be any organization or authority that verifies that the holder has a valid immunity passport to allow him to visit a building, city, conference, or a country.

Fig. 4 presents a high-level design of a blockchain-based system to create a digital medical passport to maintain the medical identity of citizens to curb the spread of COVID-19 [72]. It has implemented Ethereum-based smart contracts to minimize the medical-related frauds by presenting test results and medical information to the authorized users in a trusted, reliable, and secure manner. It has incorporated Self-diverging identity, interplanetary files system (IPFS), and Proxy reencryption to assist testing centers to provide medical passport and immunity certificates to users. Based on the medical passport, users can be allowed to travel. The authorities can exempt an individual from various social restrictions on presenting a valid digital medical passport by the user.

\section{G. Data Privacy and Self-Sovereign Identity}

Recently, many prevention and control measures for COVID-19 including stringent lockdown, remote health care, and distance-based learning are practiced globally to minimize social interactions of humans to control the spread of COVID19. The government agencies in South Korea had used personal data of their citizens including location data and credit card purchase history to track the outbreak of COVID-19. The traveling history of citizens which is identified through their location data was used to know where the citizens stayed before they were diagnosed positive against the COVID-19 test $[6,73]$. Preserving the privacy of citizen's data along with compliance with the GDPR privacy guidelines can lead to a trusted and dependable system. For many countries, the industry-standard privacy policies state that the personally identifiable information should be shared with the government agencies to assure the health safety of citizens or lawful obligation [74]. Public blockchain-based systems are highly vulnerable to data privacy breaches as they implement a zeroaccess control policy to access the blockchain network. However, being governed and managed by a single organization, private blockchain platforms are more reliable, trustworthy, and dependable to preserve the privacy of users data [74-77].

To share the health data of the citizens (e.g., COVID19's test result report) by the organizations with government agencies, organizations oftentimes sign and follow the consent 
TABLE I

THE REQUIREMENTS AND OPPORTUNITIES OF BLOCKCHAIN TECHNOLOGY TO HANDLE USE CASES RELATED TO HEALTH EMERGENCY DUE TO COVID-19 PANDEMIC.

\begin{tabular}{|c|c|c|c|c|}
\hline Application & Requirements & Blockchain Opportunities & Participants & Remarks \\
\hline $\begin{array}{l}\text { Track and Trace } \\
\text { of PPE }\end{array}$ & $\begin{array}{l}\text { - Fast identification of counterfeit } \\
\text { PPE } \\
\text { - Availability of provenance data } \\
\text { about PPE } \\
\text { - High security of PPE related data }\end{array}$ & $\begin{array}{l}\text { - Complete trace of PPE man- } \\
\text { ufacturing history } \\
\text { - Automation of PPE supply } \\
\text { chain operations } \\
\text { - Verification of authenticity of } \\
\text { PPE } \\
\text { - Transparent and fast pay- } \\
\text { ment settlements }\end{array}$ & $\begin{array}{l}\text { - PPE manufacturer } \\
\text { - PPE users } \\
\text { - Procurement man- } \\
\text { ager } \\
\text { - Distributor } \\
\text { - Quality assurance } \\
\text { manager }\end{array}$ & $\begin{array}{l}\text { - Identifying PPE related frauds re- } \\
\text { quire that all participating organiza- } \\
\text { tions should be using blockchain tech- } \\
\text { nology. } \\
\text { - Using high quality and certified PPE } \\
\text { can assist in minimizing the spread of } \\
\text { COVID-19. }\end{array}$ \\
\hline $\begin{array}{l}\text { Logistics Moni- } \\
\text { toring of Vaccine }\end{array}$ & $\begin{array}{l}\text { - Verification of genuinity of vac- } \\
\text { cine } \\
\text { - Protection of vaccine related data } \\
\text { - Guarantee of vaccine procure- } \\
\text { ment from an authorized manu- } \\
\text { facturer }\end{array}$ & $\begin{array}{l}\text { - Transparency of vaccine lo- } \\
\text { gistics operations } \\
\text { - Protection of trade documen- } \\
\text { tations } \\
\text { - Establishing data provenance } \\
\text { of substandard vaccine } \\
\text { - Prevention of compliance vi- } \\
\text { olation }\end{array}$ & $\begin{array}{l}\text { - } \text { Vaccine manufac- } \\
\text { turer } \\
\text { - Drug authority } \\
\text { - Patients } \\
\text { - Shiping agencies } \\
\text { - Quality control } \\
\text { manager } \\
\text { - Distributor } \\
\end{array}$ & $\begin{array}{l}\text { - Blockchain-based smart contracts can } \\
\text { transparently calculate the reputation } \\
\text { score for every vaccine manufacturer. } \\
\text { - Blockchain can enable relevant } \\
\text { healthcare organizations to locate and } \\
\text { buy genuine vaccines from reputed } \\
\text { organizations. }\end{array}$ \\
\hline $\begin{array}{l}\text { Incentive-Based } \\
\text { Volunteer } \\
\text { Participation } \\
\text { in Clinical Trials }\end{array}$ & $\begin{array}{l}\text { - The correctness of clinical trials } \\
\text { data } \\
\text { - Assurance of users data privacy } \\
\text { - Assurance of data access and us- } \\
\text { age in compliance with a consent } \\
\text { form }\end{array}$ & $\begin{array}{l}\text { - Assurance of compliance } \\
\text { with clinical trial rules } \\
\text { - Audit of clinical trial opera- } \\
\text { tions } \\
\text { - Consistency of clinical trial } \\
\text { data } \\
\text { - Transparency in providing } \\
\text { incentives to participants }\end{array}$ & $\begin{array}{l}\text { - Researchers } \\
\text { - Regularities } \\
\text { - Donors } \\
\text { - Drug companies }\end{array}$ & $\begin{array}{l}\text { - Blockchain technology can assist in } \\
\text { minimizing the efforts required to } \\
\text { manage clinical trial documentation. }\end{array}$ \\
\hline $\begin{array}{l}\text { Delivery of Re- } \\
\text { mote Healthcare } \\
\text { and Medical Sup- } \\
\text { plies }\end{array}$ & $\begin{array}{l}\text { - Tracking the location of medical } \\
\text { supplies } \\
\text { - Tracing the provenance of self- } \\
\text { testing COVID-19 test kits } \\
\text { - Auditability of air vehicles oper- } \\
\text { ations }\end{array}$ & $\begin{array}{l}\text { - Access to complete medical } \\
\text { history of an individual } \\
\text { - Data access and control } \\
\text { based on consent } \\
\text { management }\end{array}$ & $\begin{array}{l}\text { - Patient } \\
\text { - Aerial vehicles } \\
\text { - Pharmacy }\end{array}$ & $\begin{array}{l}\text { - Immutable blockchain technology can } \\
\text { help a buyer to view, verify, and vali- } \\
\text { date the health score of a COVID-19 } \\
\text { testing kit. }\end{array}$ \\
\hline $\begin{array}{l}\text { Digital Contact } \\
\text { Tracing }\end{array}$ & $\begin{array}{l}\text { - Assurance of data privacy } \\
\text { - Assurance of data integrity } \\
\text { - High accuracy in identifying } \\
\text { close contact with infected per- } \\
\text { son } \\
\text { - Battery friendly design of appli- } \\
\text { cation }\end{array}$ & $\begin{array}{l}\text { - Seamlessly identify individu- } \\
\text { als that have come close con- } \\
\text { tact with COVID-19 infected } \\
\text { person } \\
\text { - Automation of process to no- } \\
\text { tify the exposed individuals } \\
\text { - Real-time tracking of loca- } \\
\text { tion of individuals }\end{array}$ & $\begin{array}{ll}\text { - } & \text { Government } \\
\text { authorities } \\
\text { - Hospitals/COVID- } \\
19 \text { testing centers } \\
\text { - Individuals \& Em- } \\
\text { ployees } \\
\text { - Public/Private } \\
\text { institutes \& offices } \\
\end{array}$ & $\begin{array}{l}\text { - Existing solutions use handheld de- } \\
\text { vices such as smartphone for digital } \\
\text { contact tracing. } \\
\text { - Digital contact tracing solutions } \\
\text { should be battery and CPU resource } \\
\text { friendly. }\end{array}$ \\
\hline $\begin{array}{l}\text { Vaccination Cer- } \\
\text { tificates and Im- } \\
\text { munity Passports }\end{array}$ & $\begin{array}{l}\text { - Integrity of Vaccine certificate } \\
\text { - Assurance of data privacy } \\
\text { - Trust on COVID-19 antibody } \\
\text { testing kits }\end{array}$ & $\begin{array}{l}\text { - Identify counterfeit COVID- } \\
19 \text { antibody testing kits } \\
\text { - Identify invalid immunity } \\
\text { passport } \\
\text { - Identify valid vaccination } \\
\text { certification }\end{array}$ & $\begin{array}{l}\text { - Individuals \& Em- } \\
\text { ployee } \\
\text { - Hospitals/COVID- } \\
19 \text { testing centers } \\
\text { - Government } \\
\text { - Vaccine manufac- } \\
\text { turing company } \\
\end{array}$ & $\begin{array}{l}\text { - Blockchain can assist in assuring } \\
\text { compliance with policies to present a } \\
\text { valid immunity passport to authorities } \\
\text { visit a place/country. }\end{array}$ \\
\hline $\begin{array}{l}\text { Rapid Response } \\
\text { Registry } \\
\text { for Medical } \\
\text { Professionals }\end{array}$ & $\begin{array}{l}\text { - Registration of medical profes- } \\
\text { sionals } \\
\text { - Transparent medical resource } \\
\text { sharing } \\
\text { - Access to skilled medical profes- } \\
\text { sionals }\end{array}$ & $\begin{array}{l}\text { - Streamline hospital opera- } \\
\text { tions } \\
\text { - Verify skills of medical pro- } \\
\text { fessionals } \\
\text { - Transparent payment settle- } \\
\text { ment } \\
\text { - Verification of educational } \\
\text { certificates }\end{array}$ & $\begin{array}{ll}\text { - } & \text { Medical } \\
\text { professionals } \\
\text { - Hospitals staff }\end{array}$ & $\begin{array}{l}\text { - Blockchain can assist in availing the } \\
\text { services of medical professionals lo- } \\
\text { cating geographically at distant loca- } \\
\text { tions. }\end{array}$ \\
\hline Insurance Claims & $\begin{array}{l}\text { - Fast insurance claims settlement } \\
\text { - Low cost } \\
\text { - Fraudulent claims verification }\end{array}$ & $\begin{array}{l}\text { - Verifiability of insurance } \\
\text { claims } \\
\text { - Monetization of health data } \\
\text { - Transparent payment settle- } \\
\text { ment }\end{array}$ & $\begin{array}{l}\text { - Insurance company } \\
\text { - Patient } \\
\text { - Physicians }\end{array}$ & $\begin{array}{l}\text { - Blockchain can assist to quickly ver- } \\
\text { ify and process insurance claims of } \\
\text { COVID-19 patients. } \\
\text { - It can assist patients to share their } \\
\text { health data with insurers to monetize } \\
\text { health data. }\end{array}$ \\
\hline $\begin{array}{l}\text { Donation Track- } \\
\text { ing }\end{array}$ & $\begin{array}{l}\text { - Traceability of donation spending } \\
\text { - Fast donations processing } \\
\text { - Low operational cost }\end{array}$ & $\begin{array}{l}\text { - Verifiability of donations } \\
\text { spending } \\
\text { - Transparency in donation ac- } \\
\text { tivities } \\
\text { - Audit trials of donations }\end{array}$ & $\begin{array}{l}\text { - Donors } \\
\text { - Government } \\
\text { - Communities }\end{array}$ & $\begin{array}{l}\text { - Blockchain provides transparency in } \\
\text { activities related to funds transferring } \\
\text { and consuming that can significantly } \\
\text { increase the trust of donors. } \\
\text { - Blockchain can assist the authorities } \\
\text { to assure fair distribution of donations } \\
\text { to the communities. }\end{array}$ \\
\hline
\end{tabular}




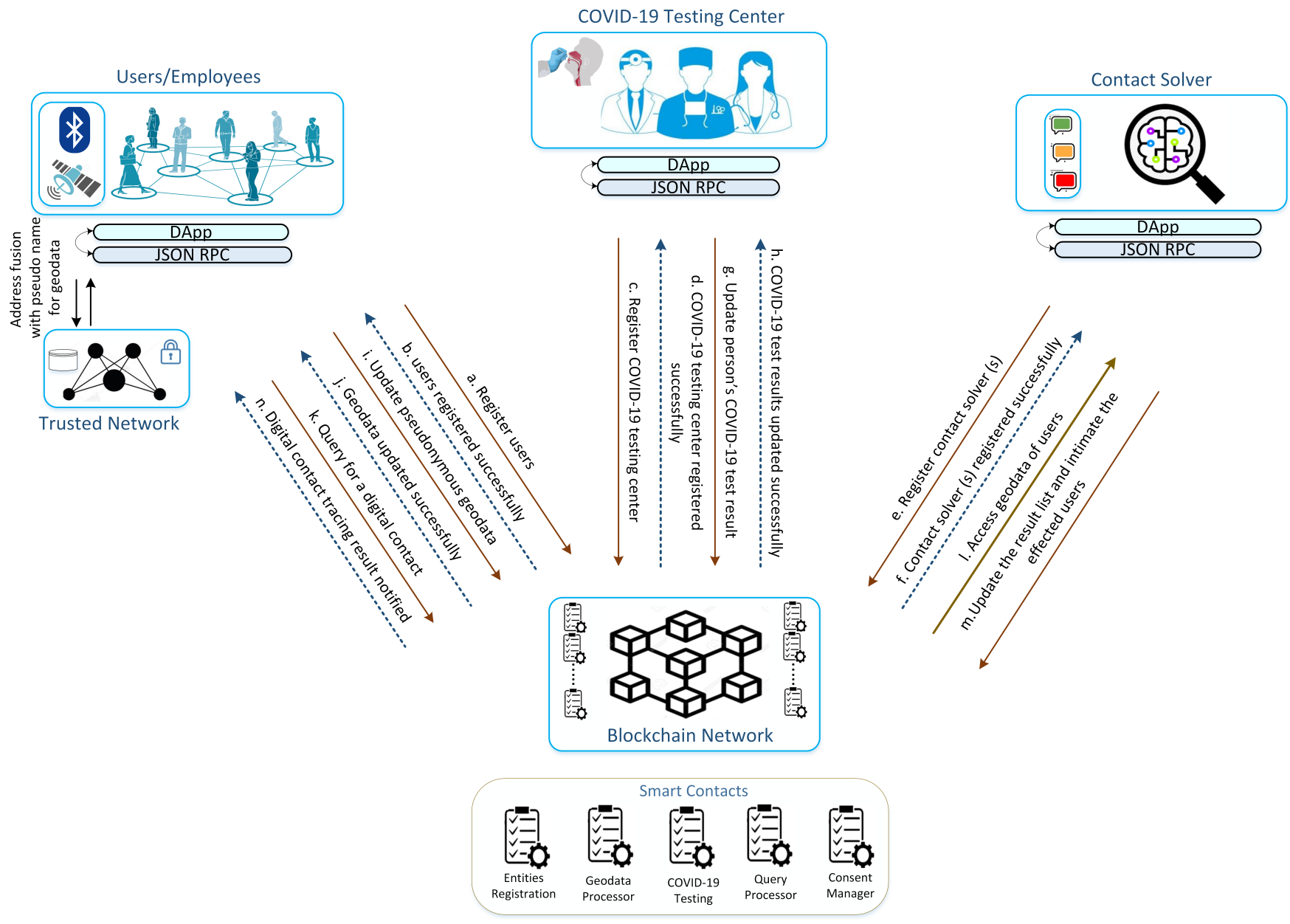

Fig. 3. Blockchain-based system for digital contact tracing to curb spreading of COVID-19.

form. A consent form outlines the rules that define the purpose of sharing user data. The traceability feature of blockchain technology can assist the authorities to audit the operations for identifying any violations or non-compliance while handling or using citizen's data. The blockchain-based smart contracts can assure that (through contact tracing) employees of an organization are immediately notified about their exposure to the COVID-19 infected patients. It assures that the credentials of virus-infected individuals are not disclosed to others to preserve data privacy. Self-sovereignty for identity management allows the users to own and control their identities without the intervening administrative authorities. It assures that an individual has full control over his data and he can allow or reject the request by the organizations to share personal health data with them [78-81]. Through self-sovereign systems, the data privacy of an individual with symptomatic symptoms of COVID-19 can be assured as the user can refuse the request of sharing personal health data with a health specialist or researcher.

\section{H. Rapid Response Registry for Medical Professionals}

Fair allocation and protection of scarce and shareable medical resources in existing public healthcare systems are challenging amid the global COVID-19 pandemic. Many studies acknowledged that the COVID-19 pandemic has overwhelmed the existing healthcare system of the world [82]. In such a scenario, rapid and immediate decisions and actions by the authorities or governments in compliance with the agreed-on policies can assure the health safety of their workers. In health systems, the making of policies for (a) allowing a caregiver to virtually visit the quarantine patients, (b) increasing hospital staff and resources, (c) operating AI-based robots to facilitate health professionals to conduct COVID-19 testing, (d) delivering medical supplies through drones, and (e) educating the community to avoid the spreading of false information about COVID-19 can assist to fight against COVID-19 pandemic $[83,84]$. In traditional healthcare systems, data about medical professionals such as doctors and nurses and resources usually sits in the silos of organizations/hospitals. As a result, it creates limited collaboration opportunities among medical professionals enrolled under different hospitals which are often located at geographically scattered locations to combat the COVID-19 pandemic [78, 85]. A rapid response registry system registers and maintains a list of medical professionals worldwide along with their role and expertise to streamline coordination among hospitals or agencies to overcome the scarcity of medical professionals.

Smart contracts deployed on the blockchain can assist in 


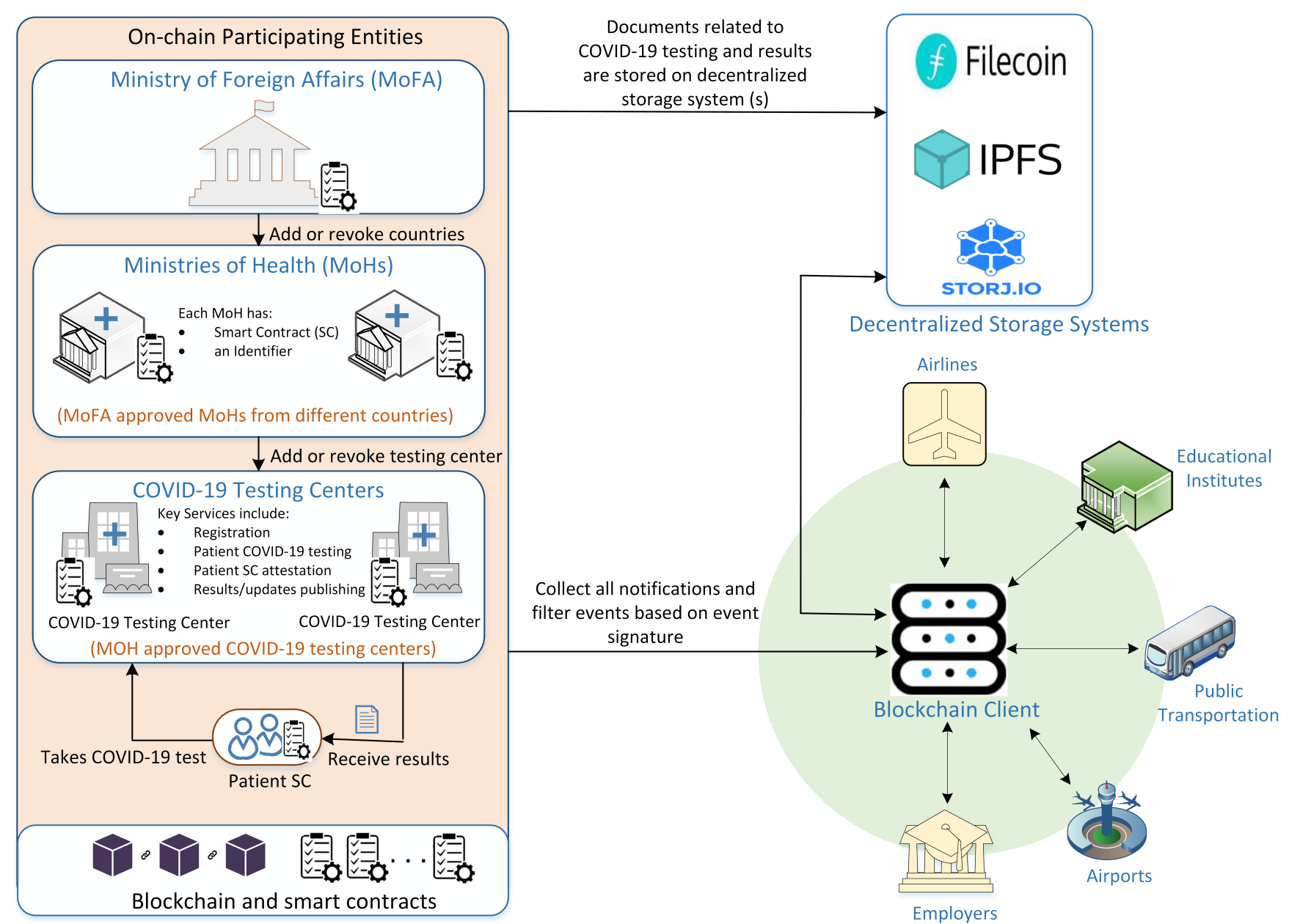

Fig. 4. High level design of a blockchain-based system for COVID-19 digital medical passports and immunity certificates.

streamlining coordinated actions of relevant healthcare organizations to efficiently identify appropriate medical professionals in a highly trustworthy and transparent way. Because of a single and unified view of the healthcare data, the authorities can identify the resource capacity, allotment, and demand of a hospital in a seamless, transparent, and trusted manner. A blockchain-based rapid response registry system can allow medical students and health professionals (employed and unemployed) to register to the blockchain platform. A smart contract can continuously monitor the data to assure that it has enough pool of reserved medical professionals to provide ondemand services to the hospitals to minimize resource scarcity issues. The on-demand services of unemployed health professionals could be either volunteer or paid (verified through a consent form). After the registration stage, smart contracts can verify the educational certificates which are shared using IPFS servers, skills that can be traced using blockchain, and other supporting documents to audit frauds. Through transparency and accountability features, it can assure that payments are settled for the services of healthcare professionals in compliance with rules inside the consent form. Similarly, through a unified and single blockchain-based health data repository, the medical professionals which are located geographically at distant locations can share data of patients with COVID-19 to enable researchers or health professionals to analyze it. Based on health data analysis, health professionals can learn and apply treatments that are effective for COVID-19 patients. For instance, analysis of data can be helpful to identify the success rate of patients treatment through blood plasma.

\section{Tracking of COVID-19 Data}

In the COVID-19 pandemic, social media has become the most widely acclaimed information sharing tool. However, due to the open nature of social networks, there is a high probability of misinformation, sensationalism, and rumors about the COVID-19 outbreak. Existing social media channels and websites are incapable to scrutinize and verify the information source $[6,9,86,87]$. As a result, fabricated or falsified data related to the COVID-19 pandemic, healthcare, or medical devices can cause panic and public confusion about who and which information sources to be trusted. It can lead to harmful self-medication and non-compliance of the public with policies designed by the government related to public movement restrictions and social distancing. Further, any prediction model or estimation of future growth of COVID19 using fabricated or falsified data will be meaningless 


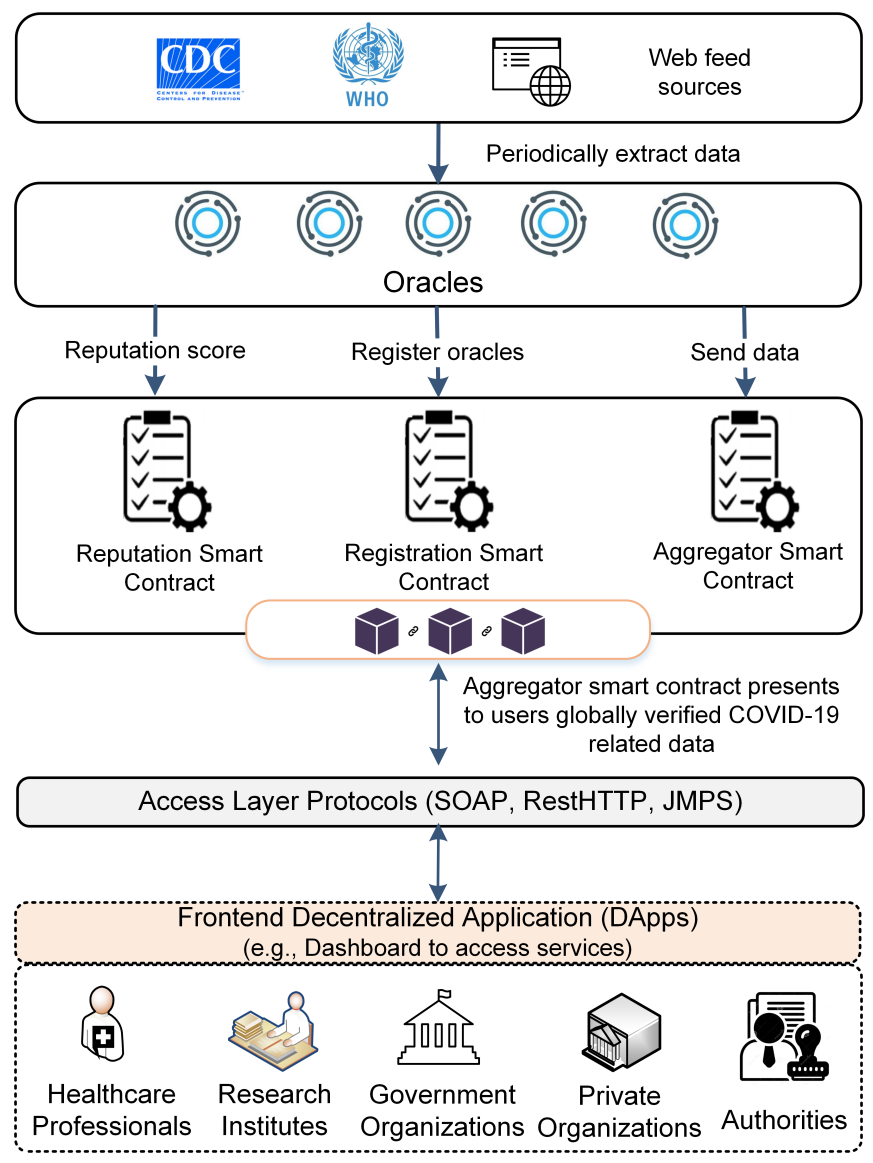

Fig. 5. Blockchain-based system for COVID-19 related data tracking through oracles and smart contracts.

$[6,31,88]$. The trustworthy data about COVID-19 can assist authorities, governments, and agencies to accurately identify infection hotspots within a geographical teriority to formulate a policy to curb the virus from spreading. Blockchain technology can successfully counter the fake information. It can identify any alteration that has been made to data by the adversaries using data provenance. It assures high reliability, transparency, integrity, and availability of COVID-19 related data for medical professionals and researchers. Thus, tracking of trusted COVID-19 data can assist authorities in improving planning and management decisions such as practicing lockdowns to isolate potentially infected territories and outbreak forecasting $[6,9,88,89]$. To identify the fake news [86], a blockchain-based system can register, rank, and filter news based on the reputation of news agencies.

Fig. 5 presents a generic blockchain-based system that can be used to track COVID-19 related data such as the development of medicines for patients with COVID-19, confirmed COVID-19 affected cases, and mortality rate in a particular country in a highly trusted and reliable manner. It employed registered oracles to fetch the COVID-19 related data from trustworthy sources such as WHO and the european center for disease control and prevention (ECDC) websites [9]. It calculates and updates the reputation score of oracles based on their performance behavior. It has implemented three smart contracts called registration, aggregator, and reputation smart contracts. Aggregator smart contract provides the latest data to the users about COVID-19. The reputation smart contract either increases or decreases the reputation score of the oracles based on their performance behavior.

\section{J. Insurance claims and Donations Tracking}

Health insurance companies can employ trusted blockchain technology to accelerate their growth and market share. The traditional insurance systems face various challenges related to fraudulent claims, complex compliance issues, high convergence time and cost in processing insurance claims, and unverifiable transaction records. In contrast to traditional health insurance systems, blockchain's accountability, transparency, and verification features can assist insurance companies to conduct audit trials of insurance claims presented by users using an immutable record of health insurance-related transactions [90]. Access to the complete health data about a COVID19 patient and complying with terms and conditions that are defined in the consent form can assist the insurers to verify the patients claim in a secure, trusted, and timely fashion. To maintain a healthy lifestyle, insurance companies can give incentives to patients by offering them tokens. Blockchain technology can assist insurance companies to verify the records of the patients to automatically transfer tokens through self-executing smart contracts [91, 92]. 
Traditional systems that are used for tracking funds donation face many challenges such as lack of transparency, trust, accountability, and traceability of donations. Blockchain technology offers a peer to peer ( $\mathrm{P} 2 \mathrm{P})$ architecture to directly transfer cryptocurrencies to the wallet of the affected people. It assists charitable organizations to verify the cryptocurrency transferring and receipts in a transparent, trusted, and accountable way. It saves time and money by eliminating the role of intermediaries to route the funds to the affected people [93, 94]. The inbuilt transparency and trust of blockchain technology can boost fundraising by charitable organizations. The traceability feature can assist the donors to verify whether their donations are utilized properly. The adaptability of blockchain by the charitable organizations can assist the government officials to fairly distribute the funds among affected citizens of a community. For instance, due to high visibility and transparency, government officials can verify the funds donated to a community of a particular geographical region. Based on the analysis, it can identify, guide, and direct the donors to a more affected community [2, 9, 93, 94].

Discussion: Blockchain technology has shown a considerable potential to enable the participating organizations to efficiently handle the COVID-19 pandemic. The adaptability of blockchain technology to reshape traditional centralizedbased healthcare systems require high data interoperability, fast transaction processing speed, and high security and privacy of the organization's data. In addition to the opportunities which are discussed above, researchers have started working to identify CVOID-19 infected individuals through the analysis of wastewater [95, 96]. Based on wastewater analysis, blockchain technology along with the AI techniques can assist the authorities to identify and predict future hotspots of the COVID-19 infected patients in a city. The rise in blood plasma donations is seen in many developing countries to treat COVID-19 patients $[97,98]$. It would be helpful to store the details about plasma donors on the blockchain to verify the effectiveness of COVID-19 treatment through blood plasma donations. However, the privacy of donors' data should be assured to avoid any criminal activity or political influence to motivate an individual to donate blood plasma. Table I summaries the aforementioned opportunities to highlight the key requirements for blockchain technology for the successful realization of each use case. It also presents the opportunities that blockchain technology can bring about for the selected application areas to introduce transparency, traceability, security, privacy, and auditability in operations. Moreover, it spots the participants that can provide or use blockchain-based services to combat COVID-19.

\section{Blockchain-based Ongoing Research Projects TO COMBAT COVID-19 PANDEMIC}

This section presents recent ongoing research projects to show how leveraging blockchain technology can help to effectively handle a public health emergency situation caused by the COVID-19 outbreak.

\section{A. Anonymous COVID-19 Testing using Epios System}

Many cases of social discrimination, abuse, and harassment are reported worldwide during COVID-19 pandemic in which individuals suffering from COVID-19 symptoms are targeted for causing pandemic and its spread. To avoid such social discrimination, hospitals or laboratories should preserve the privacy of the COVID-19 infected individuals. Epios is aimed to exploit the Telos blockchain platform to facilitate anonymous testing of individuals suffering from COVID-19. It enables the users to seamlessly connect with the laboratories which supply and process PCR testing kits. Epios assures that the payment cannot be made directly to the test processing labs. Instead, it requires the testing kit providers to provide a coupon for each testing kit to users. Further, it cryptographically protects the coupon to assist the labs to verify the payments without tracing the individual purchasing PCR testing kits. Also, Epios aims to implement a mobile application that will be used to acquire and submit the testing kits to and from the test givers in an anonymous way. The project also aims to share the COVID-19 related data such as an individual COVID-19 result and outbreak statistics with the researchers, government, and authorities while assuring that the credentials of an individual are not disclosed $[99,100]$.

\section{B. Handling Fake Infodemic using MiPasa Platform}

The COVID-19 outbreak has uncovered the dire need for a reliable, timely, secure, trusted, transparent, and privacypreserving system that should resolve the issues of existing ad-hoc, siloed, and non-scalable systems for combating COVID-19 pandemic. The verifiability of COVID-19 related data can profoundly impact the decision-making (e.g., cities lockdown) across several industries worldwide. MiPasa is a blockchain-centered platform that integrates, processes, and shares information related to the COVID-19 virus spreading from multiple verifiable sources such as WHO with registered health organizations and authorities. It helps authorities or governments in identifying both human errors and misreporting, thereby enabling data scientists and public health officials to devise solutions to limit the spread of the virus. For instance, employing data analytics on trusted and verified blockchainbased data, MiPasa can assist the state organizations to identify the COVID-19 carriers and infection hotspots in a private, secure, and timely manner. By design, MiPasa is a fully private system that is implemented on top of hyperledger fabric. Through web-based interfaces, individuals and public health representatives can use MiPasa to upload the location of the infected persons. In response, it validates it using data provided by WHO and the ECDC to assure that the new data matches the original. In the next stage, the new verified data is shared with the state authorities and health institutions that are designated by the countries [24, 101-103]. Hashlog is another blockchain-based project that provides data about the COVID19 outbreak using WHO and ECDC public data [6].

\section{Preventing Virus from Spreading using VIRI Platform}

Digital contact tracing aims to limit the spreading of airborne infectious viruses such as COVID-19. Leveraging digi- 
tal contact tracing for identifying infection hotspots through location of people can affect the user data privacy. The technological differences of the contact tracing solutions and deployment platforms can affect the adaptability and effectiveness of digital contact tracing solutions. VIRI, based on permissive blockchain technology, has filled this research gap by proposing a universal platform on a global scale for users. Developing a cross-entity platform using VIRI to track the spread of the virus in different countries can lead to identify the outbreak of COVID-19 at different places. VIRI's platform for digital contact tracing assures preserving the privacy of users' data [104]. It notifies the individuals when they made close contact with an infected person by anonymously tracking a randomly generating user identity. Later on, the individual can be alerted about infectious diseases based on the level of risk. For instance, after crossing paths with infected people, VIRI can change the status of an individual from "clear case" to a "potential infected case". Through open APIs, the VIRI platform can be integrated seamlessly with existing enterprise solutions. VIRI assures that the user has full control over his data and it is locally stored on his device. Blockchain-based stored data is anonymous (for privacy-preserving) and it can assist machine learning and AI-based tools to predict COVID19 pandemic globally $[105,106]$.

\section{Data Privacy Assurance using WIShelter Application}

WIShelter is based on WiseID application which is Wisekey's digital identity platform for providing security services to its users. Wisekey has employed blockchain technology, IoT [37], and AI-based tools to develop large scale digital identity ecosystems. WIShelter is a smartphonebased application that stores health data of individuals on the distributed ledger in a reliable and trusted manner. The records of the health data include many essential medical specimens such as allergies, blood pressure, and many other pharmaceutical details. WIShelter aims to facilitate the users to seamlessly upload their digital certificates indicating the results of the COVID-19 test on the blockchain platform [107, 108]. Through WIShelter, the uploaded COVID-19 test results of an individual can be accessed and verified by authorized government officials to issue travel permits to the individual willing for traveling. However, overlooking the privacy of users' data (e.g., COVID-19 test results) can lead to various issues related to mistreatment and discrimination with infected issues. To handle such a situation, WIShelter guarantees that the data of the users cannot be shared with others without their consent. The consent form can be duly signed by the data owner and users, and it could be transparently stored on the blockchain for accountability and auditability purposes. Moreover, to secure the medical records and data communication, WIShelter encrypts the user data. Encrypting data also assures the preservation of data privacy as stated in GDPR. WIShelter is flexible and it can assist authorities to verify the compliance with the stay at home policies designed by the authorities for COVID-19 infected patients [107-109].

\section{E. Remote Healthcare using Medicalchain and HealPoint Systems}

Telemedicine is one of the ways to prevent the spread of carnivorous through remote patient monitoring. An Ethereum and Hyperledger Fabric-based platform called Medicalchain has been used to implement remote services related to patientto-doctor consultancy and marketplace applications. It assures that health data transferring during patient-to-doctor consultancy is secure and private. Through marketplace applications, Medicalchain enables the owner of health data (patient) to privately share the data with third parties (researchers) based on an agreed-upon consent form [91]. Another platform called HealPoint enables patients to get the second opinion from a remote doctor on the health of a patient. HealPont can also recommend the most appropriate physicians to a patient based on several factors such as location, experience, and conflict of interest [110].

\section{F. Self-Sovereign Identity using COV-ID and E-Rezept Systems}

The E-Rezept platform is a remote healthcare system that is based on the principle of self-sovereign identity. It enables patients to remotely place an order for medicines by presenting their unique identifiers as proof $[111,112]$. In comparison to Medicalchain and HealPoint, E-Rezept is flexible and it can be easily integrated with existing legacy healthcare systems. $C O V-I D$ is a blockchain-based startup that aims to follow a permissioned self-sovereign identity (SSI) network called Sovrin [113]. The COV-ID system gives rewards to lawabiding citizens in an accountable and transparent manner [112]. The access pass program that was integrated with WeChat in China has employed blockchain to immutably record encrypted information related to local citizens on the distributed ledger. The data stored on the ledger was, later on, used by the authorities and governments to map a travel history of the COVID-19 infected patient. The mapping of the travel history of infected patients was used to identify infection hotspots [111, 114]. VeChain is a blockchain-based platform that supports real-time monitoring of vaccine development. It assures that the data related to vaccine development and other details such as materials and codes to package are immutable and dependable [115].

\section{OPEN RESEARCH CHALLENGES}

In this section, we briefly discuss important open research challenges along with their key causes that hinder the adoption of blockchain for COVID-19 relief. The purpose is to provide guidelines and directions to new researchers aiming to develop immediate blockchain-based solutions to battle against COVID-19.

\section{A. Cross-Platforms Communication Capabilities}

Blockchain technology can greatly improve the supply chain of PPE and vaccines by (a) allowing faster and transparent shipment of COVID-19 prevention materials, (b) enhancing traceability of shipping materials, and (c) increasing trust among participating organizations by presenting a single and 
synchronized view of shipment data. It presents a cooperative, accountable, and collaborative environment among the participating organizations including authorities, government, hospitals, and research institutes to fight with COVID-19 pandemic. The blockchain interoperability feature allows disparate blockchain-based systems to uninterruptedly communicate with each other $[22,116]$. It enables users to see, share, and access information across several blockchain platforms without requiring intermediary assistance (for translation service). Thus, the blockchain platform's interoperability support can increase the throughput, safety, and productivity of a system. It also enables a user-friendly experience among multi-users, presents a contactless and easier smart contract execution environment, provides the opportunity to develop partnerships among participating organizations, and allows smooth sharing of information [14, 116, 117]. For instance, through interoperability supported blockchain platforms, a user can perform business transactions using Bitcoin tokens on the Ethereum blockchain network. However, the diversity in technologies and differences in software designs of existing blockchain platforms are the major challenges to create an interoperable blockchain-based system $[14,116]$. The difference in supported languages, level of protection for data and transactions in the smart contracts, and recommended consensus protocols makes it challenging to propose generalized interoperability supported solutions. Moreover, an interoperable platform that hosts services for organizations that are combating the COVID-19 pandemic should provide high security, fault-tolerance, and fast transaction processing.

\section{B. Smart Contracts Security Audit}

Smart contracts implement terms and conditions of agreement among the participating organizations to automate the business processes such as tracking and tracing PPE, identifying COVID-19 hotspots through digital contact tracing, verifying vaccination certificates, and issuing immunity passports to individuals willing to travel. It automatically handles the exchange of cryptocurrencies and assets such as PPE, vaccines, and medicines in a manner that is traceable, transparent, and accountable. Despite many advantages of smart contracts such as support for automation, ease of debugging, cost efficiency, and limited human intervention required to run business processes; however, the presence of bugs inside a smart contract code can affect its normal operations that can lead to huge losses and disruptions. Being deployed on a decentralized platform, a smart contract can face several security threats from the pseudonymous malicious actors that can fully control the smart contract for malicious purposes [118-120]. Smart contracts deployed on the public blockchain platforms are often open-source. As a result, publicly accessible data and transactions on the blockchain platform can make the system highly vulnerable to malicious users. The immaturity and lack of understanding of blockchain technology can also result in flaws in the design of smart contracts. The techniques such as ContractLarva, Erays, FSolidM, EtherTrust, KEVM, and Osiris have been proposed to detect bugs and secure smart contracts against attacks of vulnerable entities $[119,121]$. Despite several efforts have been made to secure smart contracts, there is still a dire need to propose multi-objective based high-performance security protocols to further secure smart contracts.

\section{Data Privacy and Ethics}

Blockchain can greatly overcome issues of traditional clinical trial data management systems such as data inconsistency and duplication through distributed ledger technology. It stores clinical trial data in a distributed way and enables all miner nodes to possess the entire database. The open nature of the database gives rise to particular concerns of organizations about data privacy assurance and security [31, 121, 122]. Data privacy assures that the clinical trial data stored on the blockchain platform should be invisible to unauthorized individuals. It assures that health data is only shared with authorized organizations (government or authorities) and complies with the terms and conditions as defined in the consent form and GDPR privacy laws. Assuring privacy of clinical trial data through public blockchain platforms such as Bitcoin and Ethereum is challenging as data and transactions are public; whereas, private and consortium platforms such as Hyperledger fabric and Quorum are operated in a controlled environment, thereby preserving the privacy of clinical trial data. Issuing immunity passports to individuals/employees based on vaccination certification is another use case that requires data privacy assurance (from unauthorized users) to effectively eliminate the possibility of any social discrimination with COVID-19 infected patients. The techniques such as Zero-knowledge proof, attribute-based encryption (ABE), and Multi-party homomorphic obfuscations can preserve privacy of COVID-19 related data about patients [31, 66, 123]. Although the existing medical passport creation schemes have carefully preserved the privacy of COVID-19 data, there exists certain ethical issues that can affect its adaptability [122]. For example, the effect of blockchain on the environment such as carbon emission due to huge power consumption poses crucial challenges that must be given adequate attention. [20].

\section{Transaction Throughput and Network Latency}

The COVID-19 pandemic demands the traditional healthcare systems to timely and efficiently handle public health emergencies. It also requires consistent and close coordination and cooperation among organizations that are involved in health emergency operations to curb COVID-19 pandemic. The close cooperation and coordination require maintaining a consistent and synchronized record of data for streamlining operations of participating organizations to curb COVID-19. However, the amount of data generated by the organizations is very large $[16,53,82,124]$. For instance, digital contact tracing requires users to regularly monitor and update their timestamped geodata on the blockchain. Thus, the velocity of data increases that as a result creates challenges to meet fast data processing requirements of COVID-19 related affairs (healthcare). The latency of the blockchain network is mainly calculated based on the time required to mine a block. The latency can vary depending on the type of deployed blockchain platform and its specifications [31]. The high 
latency of a network results in lower transaction throughput. Ethereum, a publicly accessible platform, provides limited transaction privacy and throughput. It has a throughput of twenty transactions per second [125-127]. Private blockchain platforms are considered fast and secure and can handle up to several thousand transactions per second [34, 128, 129]. The escalating transaction rate can affect transaction fees, network energy consumption rate, and transaction processing time. The increasing transaction-rate also requires blockchain nodes to be more resource-rich to mine large-sized blocks. The incorporation of an additional edge or fog-based layer [130] in the existing frameworks for data pre-processing can help to minimize the transaction rate. Besides, communication through off-chain channels and data compression techniques can be handy to handle transaction escalating rate.

\section{E. Slow Adoption of Blockchain Technology}

COVID-19 has affected and reshaped the social life, business rules, and well-being of individuals, countries, and communities in various ways. Blockchain technology has sprung up to deal with the affairs that are related to the health of individuals by proposing digital contact tracing solutions. Digital contact tracing assures the compliance with social distancing directives which are issued by the authorities or governments to refrain the coronavirus from spreading. Further, the inherent features of blockchain technology such as traceability, decentralization, and transparency can significantly increase the trust among organizations that are involved in the manufacturing, certification, and transportation of PPE, vaccines, and medical supplies [27, 33, 127]. Blockchain technology can assist authorities, governments, and medical professionals in reviewing, recording, and tracking of demand, supplies, and logistics of epidemic prevention materials. The digitization of vaccination certification through blockchain to reopen the businesses can greatly minimize the spread of COVID-19 as fake certificates cannot be created and presented (traceability feature) by the employees while returning back to work [34, 65]. Lastly, digitizing commercial transactions through cryptocurrency can not only minimize the time and cost, but also assures seamless authentication of business processes. Despite such advantages of blockchain technology, its adaptability to combat the COVID-19 pandemic is still limited. The main reasons for the slow adoption of blockchain technology are (a) limited incentives for organizations to replace their legacy business practices, (b) lack of laws and regulations for the governance of blockchain technology, and (c) limited understanding and confidence of the users on the evolving blockchain technology. Moreover, a high energy consumption rate and complexity of mining operations of a blockchain-based system can affect its adaptability [20, 27]. As miners are often spread across various countries and continents, the incompetence of current blockchain technology to clearly define which privacy laws apply to a particular miner node can refrain organizations from adopting blockchain technology. Therefore, further research is required to propose standards, governance rules, and laws for blockchain technology to improve its adaptability by the participating organizations to combat the COVID-19 pandemic.

\section{CONCluding Remarks AND FUture RECOMMENDATIONS}

In this paper, we discussed in detail how the emerging blockchain technology features and benefits can be leveraged for combating the COVID-19 pandemic. We explored the potential blockchain applications from mainly the healthcare emergency perspective to discuss the key role that blockchain can play during the COVID-19 pandemic. We identified the key requirements of the participating organizations to develop blockchain-based systems for healthcare emergency services to combat the COVID-19 pandemic. We discussed existing blockchain-based systems that are developed recently to implement diverse services related to data privacy assurance, remote COVID-19 testing, seamless digital contact tracing, and remote outpatient health monitoring. We identified and presented several research challenges that hinder the successful implementation of blockchain technology for healthcare emergency services during the COVID-19 pandemic. Our key findings and recommendations include:

- The advantages of blockchain technology in terms of the substantial trust, security, traceability, and transparency can greatly assist the authorities to devise solutions to fight against the COVID-19 pandemic. For instance, immutable data related to the outbreak of COVID-19 in a city can be used by the authorities to correctly identify infection hotspots. Access to such crucial information can assist the authorities to formulate policies for preventing the virus from further spreading.

- Performance of digital contact tracing solutions greatly depends on the amount and velocity of collected information related to location, travel history, and COVID-19 test results of individuals. It is highly recommended that the privacy of the user's data should be preserved by the contact tracing solutions.

- Blockchain technology is intended to provide a cooperative, accountable, and collaborative environment for participants that are involved in the supply chain logistics of PPE or vaccine. The adoption rate of blockchain technology by participants greatly depends on the operational transparency and assurance of compliance with a regulation to protect data against its misuse.

- Permissioned blockchain platforms such as Hyperledger fabric are well suited for digitizing services to develop the COVID-19 vaccine and issue immunity passport to individuals who want to travel. Considering the user requirements of such services, there is a great need to develop lightweight blockchain platforms that should offer better performance.

- Compliance with GDPR laws for employing blockchain technology to access and use public health and location data by the government agencies to make policies for the health safety of people is challenging. Such limitations can greatly affect the adaptability of blockchain technology. 


\section{ACKNOWLEDGMENT}

This publication is based upon work supported by the Khalifa University of Science and Technology under Awards No. CIRA-2019-001 and RCII-2019-002, Center for Digital Supply Chain and Operations Management.

\section{REFERENCES}

[1] Q.-V. Pham, D. C. Nguyen, W.-J. Hwang, P. N. Pathirana, et al., "Artificial intelligence (AI) and big data for coronavirus (COVID-19) pandemic: A survey on the state-of-the-arts," IEEE Access, vol. 8, pp. 130820 $130839,2020$.

[2] V. Chamola, V. Hassija, V. Gupta, and M. Guizani, "A comprehensive review of the COVID-19 pandemic and the role of IoT, Drones, AI, Blockchain, and 5G in managing its impact," IEEE Access, vol. 8, pp. $90225-$ $90265,2020$.

[3] L. Kang, Y. Li, S. Hu, M. Chen, C. Yang, B. X. Yang, Y. Wang, J. Hu, J. Lai, X. Ma, et al., "The mental health of medical workers in Wuhan, China dealing with the 2019 novel coronavirus," The Lancet Psychiatry, vol. 7, no. 3, p. e14, 2020.

[4] James K. Jackson, "Global economic effects of COVID19 ," [Online]. Accessed on 12/08/2020, August 2020, https://fas.org/sgp/crs/row/R46270.pdf.

[5] Christina Maxouris, "WHO coronavirus disease (COVID-19) dashboard ," [Online]. Accessed on 12/08/2020, August 2020, https://covid19.who.int/.

[6] D. Nguyen, M. Ding, P. N. Pathirana, and A. Seneviratne, "Blockchain and AI-based solutions to combat coronavirus (COVID-19)like epidemics: A survey," TechRxiv, DOI: 10.20944/preprints202004.0325.v1, 2020.

[7] H. Choudhury, B. Goswami, and S. K. Gurung, "Covidchain: An anonymity preserving blockchain based framework for protection against Covid-19," arXiv preprint arXiv:2005.10607, 2020.

[8] N. Ahmed, R. A. Michelin, W. Xue, S. Ruj, R. Malaney, S. S. Kanhere, A. Seneviratne, W. Hu, H. Janicke, and S. Jha, "A survey of COVID-19 contact tracing Apps," arXiv preprint arXiv:2006.10306, 2020.

[9] D. Marbouh, T. Abbasi, F. Maasmi, I. Omar, M. Debe, K. Salah, R. Jayaraman, and S. Ellahham, "Blockchain for COVID-19: Review, opportunites and a trusted tracking system," TechRxiv. Preprint. https://doi.org/10.36227/techrxiv.12609344.v2, 2020.

[10] H. Xu, L. Zhang, O. Onireti, Y. Fang, W. B. Buchanan, and M. A. Imran, "Beeptrace: Blockchain-enabled privacy-preserving contact tracing for COVID-19 pandemic and beyond," arXiv preprint arXiv:2005.10103, 2020.

[11] J. Bay, J. Kek, A. Tan, C. S. Hau, L. Yongquan, J. Tan, and T. A. Quy, "Bluetrace: A privacy-preserving protocol for community-driven contact tracing across borders," Government Technology Agency-Singapore, Tech. Rep, 2020.
[12] M. H. Fitzgerald and P. A. Phillips, "Centralized and non-centralized ethics review: A five nation study," Accountability in Research, vol. 13, no. 1, pp. 47-74, 2006.

[13] S. Kungpisdan, "Accountability in centralized payment environments," in 9th International Symposium on Communications and Information Technology. IEEE, 2009, pp. 1022-1027.

[14] M. Torky and A. E. Hassanien, "COVID-19 blockchain framework: Innovative approach," arXiv preprint arXiv:2004.06081, 2020.

[15] L. Bach, B. Mihaljevic, and M. Zagar, "Comparative analysis of blockchain consensus algorithms," in 41 st International Convention on Information and Communication Technology, Electronics and Microelectronics (MIPRO). IEEE, 2018, pp. 1545-1550.

[16] K. Yeow, A. Gani, R. W. Ahmad, J. J. Rodrigues, and K. Ko, "Decentralized consensus for edge-centric internet of things: A review, taxonomy, and research issues," IEEE Access, vol. 6, pp. 1513-1524, 2017.

[17] Y. Zhen, M. Yue, C. Zhong-yu, T. Chang-bing, and C. Xin, "Zero-determinant strategy for the algorithm optimize of blockchain PoW consensus," in 36th Chinese Control Conference (CCC). IEEE, 2017, pp. 14411446.

[18] D. Vujičić, D. Jagodić, and S. Ranić, "Blockchain technology, Bitcoin, and Ethereum: A brief overview," in 17th international symposium infoteh-jahorina (infoteh). IEEE, 2018, pp. 1-6.

[19] C. Cachin et al., "Architecture of the Hyperledger blockchain fabric," in Workshop on distributed cryptocurrencies and consensus ledgers, vol. 310, no. 4, 2016.

[20] V. Denisova, A. Mikhaylov, and E. Lopatin, "Blockchain infrastructure and growth of global power consumption," International Journal of Energy Economics and Policy, vol. 9, no. 4, p. 22, 2019.

[21] I. Radanović and R. Likić, "Opportunities for use of blockchain technology in medicine," Applied health economics and health policy, vol. 16, no. 5, pp. 583590, 2018.

[22] Carlo R.W. De Meijer, "Blockchain and interoperability: key to mass adoption ," [Online]. Accessed on 12/08/2020, July 2020, https://www.finextra.com/blogposting/18972/blockchainand-interoperability-key-to-mass-adoption.

[23] A. Azim, M. N. Islam, and P. E. Spranger, "Blockchain and novel coronavirus: Towards preventing COVID-19 and future pandemics," American Journal of Medicine, no. Ahead of Print, 2020.

[24] Susan Miller, "Building a blockchain to verify COVID-19 data ," [Online]. Accessed on 03/08/2020, April 2020, https://gcn.com/articles/2020/04/06/mipasablockchain-covid-tracking.aspx.

[25] E. Livingston, A. Desai, and M. Berkwits, "Sourcing personal protective equipment during the COVID-19 pandemic," Jama, vol. 323, no. 19, pp. 1912-1914, 2020 . 
[26] H. Bauchner, P. B. Fontanarosa, and E. H. Livingston, "Conserving supply of personal protective equipment," Europe PMC, vol. 323, no. 19, pp. 1911-1911, 2020.

[27] S. Nagesh and S. Chakraborty, "Saving the frontline health workforce amidst the COVID-19 crisis: Challenges and recommendations," Journal of Global Health, vol. 10, no. 1, 2020.

[28] N. J. Rowan and J. G. Laffey, "Challenges and solutions for addressing critical shortage of supply chain for personal and protective equipment (PPE) arising from coronavirus disease (COVID19) pandemic-case study from the republic of ireland," Science of The Total Environment, p. 138532, 2020.

[29] F. Girardi, G. De Gennaro, L. Colizzi, and N. Convertini, "Improving the healthcare effectiveness: The possible role of EHR, IoMT and Blockchain," Electronics, vol. 9, no. 6, p. 884, 2020.

[30] K. A. Clauson, E. A. Breeden, C. Davidson, and T. K. Mackey, "Leveraging blockchain technology to enhance supply chain management in healthcare: An exploration of challenges and opportunities in the health supply chain," Blockchain in Healthcare Today, vol. 1, no. 3, pp. 1-12, 2018.

[31] A. Kalla, T. Hewa, R. A. Mishra, M. Ylianttila, and M. Liyanage, "The role of blockchain to fight against COVID-19," IEEE Engineering Management Review, 2020.

[32] B. Yong, J. Shen, X. Liu, F. Li, H. Chen, and Q. Zhou, "A blockchain based system for safe vaccine supply and supervision," arXiv preprint arXiv:2004.06081.

[33] L. J. Ramirez Lopez and N. Beltrán Álvarez, "Blockchain application in the distribution chain of the COVID-19 vaccine: A designing under study," 2020.

[34] B. Yong, J. Shen, X. Liu, F. Li, H. Chen, and Q. Zhou, "An intelligent blockchain-based system for safe vaccine supply and supervision," International Journal of Information Management, vol. 52, p. 102024, 2020.

[35] C. Fu, "Milestone and challenges: Lessons from defective vaccine incidents in China," Human Vaccines \& Immunotherapeutics, vol. 16, no. 1, pp. 80-80, 2020.

[36] L. F. Peysson, "Tracing and control of raw materials sourcing for vaccine manufacturers," Biologicals, vol. 38, no. 3, pp. 352-353, 2010.

[37] K. Salah, A. Alfalasi, M. Alfalasi, M. Alharmoudi, M. Alzaabi, A. Alzyeodi, and R. Ahmad, "IoT-enabled shipping container with environmental monitoring and location tracking," in 2020 IEEE 17th Annual Consumer Communications \& Networking Conference (CCNC). IEEE, 2020, pp. 1-6.

[38] M. Benchoufi and P. Ravaud, "Blockchain technology for improving clinical research quality," Trials, vol. 18, no. 1, pp. 1-5, 2017.

[39] I. Omar, R. Jayaraman, K. Salah, I. Yaqoob, and S. Ellahham, "Applications of blockchain technology in clinical trials: Review and open challenges," TechRxiv. Preprint. https://doi.org/10.36227/techrxiv.12635783.v1, 2020.

[40] T. Hirano, T. Motohashi, K. Okumura, K. Takajo,
T. Kuroki, D. Ichikawa, Y. Matsuoka, E. Ochi, and T. Ueno, "Data validation and verification using blockchain in a clinical trial for breast cancer: Regulatory sandbox," Journal of Medical Internet Research, vol. 22, no. 6, p. e18938, 2020.

[41] K. Thorlund, L. Dron, J. Park, G. Hsu, J. I. Forrest, and E. J. Mills, "A real-time dashboard of clinical trials for COVID-19," The Lancet Digital Health, vol. 2, no. 6, pp. e286-e287, 2020.

[42] S. L. Bernstein and J. Feldman, "Incentives to participate in clinical trials: Practical and ethical considerations," The American Journal of Emergency Medicine, vol. 33, no. 9, pp. 1197-1200, 2015.

[43] Suhail Chughtai, Samer Ellahham, "Telemedicine to revolutionize outpatient based healthcare," [Online]. Accessed on 31/05/2020, May 2020, http://thearabhospital.com/articles-eng/telemedicinerevolutionize-outpatient-based-healthcare/.

[44] Jeff Gorke, "Telehealth continues to change the face of healthcare delivery - For the better," [Online]. Accessed on 27/04/2020, April 2020, https://www.forbes.com/sites/jeffgorke/2019/09/24/telehealthcontinues-to-change-the-face-of-healthcare-deliveryfor-the-better/58669cbf565f.

[45] A. F. da Conceição, F. S. C. da Silva, V. Rocha, A. Locoro, and J. M. Barguil, "Eletronic health records using blockchain technology," arXiv preprint arXiv:1804.10078, 2018.

[46] S. Jarrett, L. Yang, and S. Pagliusi, "Roadmap for strengthening the vaccine supply chain in emerging countries: Manufacturers' perspectives," Vaccine: $X$, vol. 5, 2020.

[47] R. Kumar and R. Tripathi, "Traceability of counterfeit medicine supply chain through blockchain," in 2019 11th International Conference on Communication Systems \& Networks (COMSNETS). IEEE, 2019, pp. 568570.

[48] J. Yang. Three ways china is using drones to fight coronavirus. Accessed on: July 19, 2020. [Online]. Available: https://www.weforum.org/agenda/2020/03/ threeways-china-is-using-drones-to-fight-coronavirus

[49] B. Zhu, L. Xie, D. Han, X. Meng, and R. Teo, "A survey on recent progress in control of swarm systems," Science China Information Sciences, vol. 60, no. 7, p. 070201, 2017.

[50] W. H. Organization et al., "Digital tools for COVID19 contact tracing: Contact tracing in the context of COVID-19," World Health Organization, Tech. Rep., 2020.

[51] L. Ferretti, C. Wymant, M. Kendall, L. Zhao, A. Nurtay, L. Abeler-Dörner, M. Parker, D. Bonsall, and C. Fraser, "Quantifying SARS-CoV-2 transmission suggests epidemic control with digital contact tracing," Science, vol. 368, no. 6491, 2020.

[52] H. Cho, D. Ippolito, and Y. W. Yu, "Contact tracing mobile Apps for COVID-19: Privacy considerations and related trade-offs," arXiv preprint arXiv:2003.11511, 2020. 
[53] M. Salathé, C. L. Althaus, R. Neher, S. Stringhini, E. Hodcroft, J. Fellay, M. Zwahlen, G. Senti, M. Battegay, A. Wilder-Smith, et al., "COVID-19 epidemic in Switzerland: On the importance of testing, contact tracing and isolation." Swiss Medical Weekly, vol. 150, no. 11-12, p. w20225, 2020.

[54] L. Reichert, S. Brack, and B. Scheuermann, "Privacypreserving contact tracing of COVID-19 patients," IACR Cryptol. ePrint Arch., vol. 2020, p. 375, 2020.

[55] S. Wang, S. Ding, and L. Xiong, "A new system for surveillance and digital contact tracing for COVID19: Spatiotemporal reporting over network and GPS," JMIR, vol. 8, no. 6, p. e19457, 2020.

[56] M. M. Arifeen, A. Al Mamun, M. S. Kaiser, and M. Mahmud, "Blockchain-enable contact tracing for preserving user privacy during COVID-19 outbreak," Preprints. 2020070502 (doi: 10.20944/preprints202007.0502.v1), 2020.

[57] W. Lv, S. Wu, C. Jiang, Y. Cui, X. Qiu, and Y. Zhang, "Decentralized blockchain for privacy-preserving largescale contact tracing," arXiv preprint arXiv:2007.00894, 2020.

[58] Q. Tang, "Privacy-preserving contact tracing: Current solutions and open questions," arXiv preprint arXiv:2004.06818, 2020.

[59] A. Chawla and S. Ro, "Coronavirus (COVID-19)-Is blockchain a true savior in this pandemic crisis," Available at SSRN 3655337, 2020.

[60] J. K. Liu, M. H. Au, T. H. Yuen, C. Zuo, J. Wang, A. Sakzad, X. Luo, and L. Li, "Privacy-preserving covid-19 contact tracing App: A zero-knowledge proof approach," IACR Cryptol. EPrint Arch., vol. 2020, p. 528, 2020.

[61] D. Leith and S. Farrell, "Gaen due diligence: Verifying the Google/Apple COVID exposure notification API," 2020.

[62] M. Torky and A. E. Hassanien, "Covid-19 blockchain framework: Innovative approach," arXiv preprint arXiv:2004.06081, 2020.

[63] R. Kumar, A. A. Khan, S. Zhang, W. Wang, Y. Abuidris, W. Amin, and J. Kumar, "Blockchain-federated-learning and deep learning models for covid-19 detection using ct imaging," arXiv preprint arXiv:2007.06537, 2020.

[64] W. F. Marshall. How do COVID-19 antibody tests differ from diagnostic tests? Accessed on 8/8/2020. [Online]. Available: https: //www.mayoclinic.org/diseases-conditions/coronavirus/ expert-answers/covid-antibody-tests/faq-20484429

[65] M. Eisenstadt, M. Ramachandran, N. Chowdhury, A. Third, and J. Domingue, "Covid-19 antibody test/vaccination certification: There's an app for that," Journal of Engineering in Medicine and Biology, vol. 1, pp. 148-155, 2020.

[66] A. Bansal, C. Garg, and R. P. Padappayil, "Optimizing the implementation of COVID-19 "immunity certificates" using blockchain," Journal of Medical Systems, vol. 44, no. 9, pp. 1-2, 2020.

[67] C. Hicks, D. Butler, C. Maple, and J. Crowcroft, "Se- cureabc: Secure antibody certificates for COVID-19," arXiv preprint arXiv:2005.11833, 2020.

[68] A. Mohsin, A. Zaidan, B. Zaidan, O. Albahri, A. Albahri, M. Alsalem, and K. Mohammed, "Based blockchain-PSO-AES techniques in finger vein biometrics: A novel verification secure framework for patient authentication," Computer Standards \& Interfaces, vol. 66, p. 103343, 2019.

[69] Allexandra L Phelan, "COVID-19 immunity passports and vaccination certificates: Scientific, equitable, and legal challenges," [Online]. Accessed on 24/08/2020, May 2020, https://www.thelancet.com/journals/lancet/article/PIIS01406736(20)31034-5/fulltext.

[70] H.-N. Dai, M. Imran, and N. Haider, "Blockchainenabled Internet of Medical Things to combat COVID19," arXiv preprint arXiv:2008.09933, 2020.

[71] C. Marios Angelopoulos, A. Damianou, and V. Katos, "DHP framework: Digital health passports using blockchain-Use case on international tourism during the COVID-19 pandemic," arXiv e-prints, pp. arXiv-2005, 2020.

[72] H. R. Hasan, K. Salah, R. Jayaraman, J. Arshad, I. Yaqoob, M. Omar, and S. Ellahham, "Blockchainbased solution for COVID-19 digital medical passports and immunity certificates," TechRxiv. Preprint. https://doi.org/10.36227/techrxiv.12800360.v1, 2020.

[73] M. Alblooshi, K. Salah, and Y. Alhammadi, "Blockchain-based ownership management for medical IoT (MIoT) devices," in International Conference on Innovations in Information Technology (IIT). IEEE, 2018, pp. 151-156.

[74] Simona Rollinson, "COVID-19's impact on data privacy, protection and security," [Online]. Accessed on 20/07/2020, 2020, https://www.episerver.com/guides/covid-19-privacyconsiderations.

[75] M. Ienca and E. Vayena, "On the responsible use of digital data to tackle the COVID-19 pandemic," Nature Medicine, vol. 26, no. 4, pp. 463-464, 2020.

[76] S. Keesara, A. Jonas, and K. Schulman, "COVID19 and health care's digital revolution," New England Journal of Medicine, vol. 382, no. 23, p. e82, 2020.

[77] J. Abeler, M. Bäcker, U. Buermeyer, and H. Zillessen, "COVID-19 contact tracing and data protection can go together," JMIR, vol. 8, no. 4, p. e19359, 2020.

[78] Don Tapscott, "A new technology to combat the pandemic," [Online]. Accessed on 14/07/2020, 2020, https://thinkers50.com/blog/a-new-technology-tocombat-the-pandemic/.

[79] B. Faber, G. C. Michelet, N. Weidmann, R. R. Mukkamala, and R. Vatrapu, "BPDIMS: A blockchain-based personal data and identity management system," in Proceedings of the 52nd Hawaii International Conference on System Sciences, 2019.

[80] Y. Liu, D. He, M. S. Obaidat, N. Kumar, M. K. Khan, K.-K. R. Choo, et al., "Blockchain-based identity management systems: A review," Journal of Network 
and Computer Applications, p. 102731, 2020.

[81] L. Lesavre, P. Varin, P. Mell, M. Davidson, and J. Shook, "A taxonomic approach to understanding emerging blockchain identity management systems," National Institute of Standards and Technology, Tech. Rep., 2019.

[82] C. M. Cutter, C. Nelson, and M. Abir, "Accountability to population health in the COVID-19 pandemic: Designing health care delivery within a social responsibility framework," Population Health Management, 2020.

[83] Niamh McKenna, "COVID-19: Rapid response in healthcare ," [Online]. Accessed on 22 July 2020, March 2020, https://www.accenture.com/gben/insights/health/.

[84] D. Resiere, D. Resiere, and H. Kallel, "Implementation of medical and scientific cooperation in the Caribbean using blockchain technology in coronavirus (COVID19) pandemics," Journal of Medical Systems, vol. 44, pp. 1-2, 2020.

[85] Nigel Gopie, "Blockchain's role in COVID19 response and recovery ," [Online]. Accessed on 22/07/2020, April 2020, https://www.ibm.com/blogs/blockchain/2020/04/blockchainsrole-in-covid-19-response-and-recovery/.

[86] G. Pennycook, J. McPhetres, Y. Zhang, J. G. Lu, and D. G. Rand, "Fighting COVID-19 misinformation on social media: Experimental evidence for a scalable accuracy-nudge intervention," Psychological science, vol. 31, no. 7, pp. 770-780, 2020.

[87] O. D. Apuke and B. Omar, "Fake news and COVID-19: Modelling the predictors of fake news sharing among social media users," Telematics and Informatics, p. 101475, 2020.

[88] Elliot Smith, "Iran minister accuses 'some countries' of not declaring their coronavirus cases," [Online]. Accessed on 22/07/2020, March 2020, https://www.cnbc.com/2020/03/06/iran-ministeraccuses-some-countries-of-not-declaring-coronaviruscases.html.

[89] P. Fraga-Lamas and T. M. Fernández-Caramés, "Fake news, disinformation, and deepfakes: Leveraging distributed ledger technologies and blockchain to combat digital deception and counterfeit reality," IT Professional, vol. 22, no. 2, pp. 53-59, 2020.

[90] R. B. Fekih and M. Lahami, "Application of blockchain technology in healthcare: A comprehensive study," in International Conference on Smart Homes and Health Telematics. Springer, 2020, pp. 268-276.

[91] "Medicalchain," White Paper, Medicalchain SA, March 2018.

[92] M. Raikwar, S. Mazumdar, S. Ruj, S. S. Gupta, A. Chattopadhyay, and K.-Y. Lam, "A blockchain framework for insurance processes," in 9th IFIP International Conference on New Technologies, Mobility and Security (NTMS). IEEE, 2018, pp. 1-4.

[93] D. Jayasinghe, S. Cobourne, K. Markantonakis, R. N. Akram, and K. Mayes, "Philanthropy on the blockchain," in IFIP International Conference on Information Security Theory and Practice. Springer, 2017, pp. $25-38$.

[94] A. Singh, R. Rajak, H. Mistry, and P. Raut, "Aid, Charity and donation tracking system using blockchain," in 2020 4th International Conference on Trends in Electronics and Informatics (ICOEI)(48184). IEEE, 2020, pp. 457-462.

[95] W. Ahmed, N. Angel, J. Edson, K. Bibby, A. Bivins, J. W. O'Brien, P. M. Choi, M. Kitajima, S. L. Simpson, J. Li, et al., "First confirmed detection of SARS-CoV2 in untreated wastewater in Australia: A proof of concept for the wastewater surveillance of COVID-19 in the community," Science of The Total Environment, p. 138764, 2020.

[96] M. Kumar, A. K. Patel, A. V. Shah, J. Raval, N. Rajpara, M. Joshi, and C. G. Joshi, "First proof of the capability of wastewater surveillance for COVID-19 in india through detection of genetic material of SARS-CoV-2," Science of The Total Environment, p. 141326, 2020.

[97] C. Shen, Z. Wang, F. Zhao, Y. Yang, J. Li, J. Yuan, F. Wang, D. Li, M. Yang, L. Xing, et al., "Treatment of 5 critically ill patients with COVID-19 with convalescent plasma," Jama, vol. 323, no. 16, pp. 1582-1589, 2020.

[98] K. Duan, B. Liu, C. Li, H. Zhang, T. Yu, J. Qu, M. Zhou, L. Chen, S. Meng, Y. Hu, et al., "Effectiveness of convalescent plasma therapy in severe COVID-19 patients," Proceedings of the National Academy of Sciences, vol. 117, no. 17, pp. 9490-9496, 2020.

[99] "Anonymous epidemic disease testing," White Paper, Douglas Horn, 2020.

[100] Charles Brett, "Telos launches Epios project for anonymous COVID-19 testing," [Online]. Accessed on 02/08/2020, July 2020, https://www.enterprisetimes.co.uk/.

[101] Susan Miller, "MiPasa: An open data platform to support COVID-19 response ," [Online]. Accessed on 03/08/2020, March 2020, https://mipasa.org/blog/mipasa-an-open-data-platformto-support-covid-19-response/.

[102] Jonathan Levi, Porter Stowell, "Utilize blockchainbacked COVID-19 data with MiPasa by HACERA ," [Online]. Accessed on 03/08/2020, May 2020, https://app.mipasa.org/docs/call-for-code-2020.

[103] Denise Hines, "Blockchain and the COVID19 pandemic: How evolving societal need accelerates emerging technologies ," [Online]. Accessed on 19/08/2020, May 2020, https://www.himss.org/news/blockchain-coronavirusemerging-technologies.

[104] Sam brake guia, "VIRI creates global anonymous contact-tracing platform to stop spread of COVID19 ," [Online]. Accessed on 18/08/2020, June 2020, https://startupbeat.com/.

[105] Jacob Crompton, "VIRI creates global anonymous contact-tracing platform to stop spread of COVID19 ," [Online]. Accessed on 04/08/2020, June 2020, https://www.viri.io/.

[106] Andy Moss, Connor Spelliscy, John Borthwick, 
"Demonstrating 15 contact tracing and other tools built to mitigate the impact of COVID-19 ," [Online]. Accessed on 04/08/2020, June 2020, https://techcrunch.com/2020/06/05/.

[107] Carlos Moreira,Lena Cati, “WISeKey's cuttingedge wise-authentic identity blockchain technology ," [Online]. Accessed on 04/08/2020, Oct 2019, https://www.wisekey.com/press/.

[108] Carlos Moreira, "Managed PKI services," [Online]. Accessed on 04/08/2020, March 2020, https://www.wisekey.com/products-services/digitalidentity-pki/trust-services/managed-pki-services/.

[109] Carlos Moreira A, "WISeKey's WIShelter App now includes a health digital certificate allowing users to share their medical status via a secure QR-CODE ," [Online]. Accessed on 04/08/2020, June 2020, https://www.newsbreak.com/news/.

[110] "Healpoint," White Paper, Laboratory Information Systems,Los Angeles, 2018.

[111] Ahmed El Sherif, "Spherity contributes to Hackathon for coronavirus solutions ," [Online]. Accessed on 06/08/2020, June 2020, https://medium.com/spherity/spherity-contributesto-wirvsvirus-hackathon-for-corona-virus-solutions3c59b1557fdf.

[112] Chirag "Bhardwaj, "12 Tell-tale signs of blockchain adoption amidst COVID-19," [Online]. Accessed on 06/08/2020, July 2020, https://appinventiv.com/blog/blockchain-adoptionamidst-coronavirus/.

[113] C. Saraf and S. Sabadra, "Blockchain platforms: A compendium," in 2018 IEEE International Conference on Innovative Research and Development (ICIRD). IEEE, 2018, pp. 1-6.

[114] Hua Xia, "Blockchain technology improves coronavirus response," [Online]. Accessed on 06/08/2020, Feb 2020, http://www.xinhuanet.com/english/202002/17/c138791795.

[115] Jill mandrino, "VeChain announces blockchain vaccine tracing solution for China," [Online]. Accessed on 31/08/2020, August 2018, https://www.nasdaq.com/articles/vechain-announcesblockchain-vaccine-tracing-solution-china-2018-08-16.

[116] M. Vukolic, "On the interoperability of decentralized exposure notification systems," arXiv preprint arXiv:2006.13087, 2020.

[117] A. Khatoon, "Use of blockchain technology to curb novel coronavirus disease (COVID-19) transmission," Available at SSRN 3584226, 2020.

[118] Y. Huang, Y. Bian, R. Li, J. L. Zhao, and P. Shi, "Smart contract security: A software lifecycle perspective," IEEE Access, vol. 7, pp. 150 184-150 202, 2019.

[119] S. Sayeed, H. Marco-Gisbert, and T. Caira, "Smart contract: Attacks and protections," IEEE Access, vol. 8, pp. 24 416-24 427, 2020.

[120] Z. Gao, L. Jiang, X. Xia, D. Lo, and J. Grundy, "Checking smart contracts with structural code embedding," Transactions on Software Engineering, 2020.
[121] M. Di Angelo and G. Salzer, "A survey of tools for analyzing Ethereum smart contracts," in International Conference on Decentralized Applications and Infrastructures (DAPPCON). IEEE, 2019, pp. 69-78.

[122] T. C. Voo, H. Clapham, and C. C. Tam, "Ethical implementation of immunity passports during the COVID-19 pandemic," The Journal of Infectious Diseases, vol. 222, no. 5, pp. 715-718, 2020.

[123] D. S. W. Ting, L. Carin, V. Dzau, and T. Y. Wong, "Digital technology and COVID-19," Medicine, vol. 26, no. 4, pp. 459-461, 2020.

[124] J. E. Hollander and B. G. Carr, "Virtually perfect? Telemedicine for COVID-19," Journal of Medicine, vol. 382, no. 18, pp. 1679-1681, 2020.

[125] G. Wood et al., "Ethereum: A secure decentralised generalised transaction ledger," Ethereum Project Yellow Paper, vol. 151, no. 2014, pp. 1-32, 2014.

[126] R. Böhme, N. Christin, B. Edelman, and T. Moore, "Bitcoin: Economics, technology, and governance," Journal of Economic Perspectives, vol. 29, no. 2, pp. 213-38, 2015.

[127] M. Muzammal, Q. Qu, and B. Nasrulin, "Renovating blockchain with distributed databases: An open source system," Future Generation Computer Systems, vol. 90, pp. 105-117, 2019.

[128] S. Pongnumkul, C. Siripanpornchana, and S. Thajchayapong, "Performance analysis of private blockchain platforms in varying workloads," in 26th International Conference on Computer Communication and Networks (ICCCN). IEEE, 2017, pp. 1-6.

[129] L. Fuentes, "ClinicAppChain: A low-cost blockchain Hyperledger solution for healthcare," in International Congress on Blockchain and Applications, vol. 1010. Springer, 2019, p. 36.

[130] M. Debe, K. Salah, M. H. U. Rehman, and D. Svetinovic, "IoT public fog nodes reputation system: A decentralized solution using Ethereum blockchain," IEEE Access, vol. 7, pp. 178 082-178 093, 2019. 